\title{
Rate-cost tradeoffs in control
}

\author{
Victoria Kostina, Babak Hassibi
}

\begin{abstract}
Consider a control problem with a communication channel connecting the observer of a linear stochastic system to the controller. The goal of the controller is to minimize a quadratic cost function in the state variables and control signal, known as the linear quadratic regulator (LQR). We study the fundamental tradeoff between the communication rate $r$ bits/sec and the expected cost $b$. We obtain a lower bound on a certain rate-cost function, which quantifies the minimum directed mutual information between the channel input and output that is compatible with a target $L Q R$ cost. The rate-cost function has operational significance in multiple scenarios of interest: among others, it allows us to lower-bound the minimum communication rate for fixed and variable length quantization, and for control over noisy channels. We derive an explicit lower bound to the rate-cost function, which applies to the vector, non-Gaussian, and partially observed systems, thereby extending and generalizing an earlier explicit expression for the scalar Gaussian system, due to Tatikonda el al. [2]. The bound applies as long as the differential entropy of the system noise is not $-\infty$. It can be closely approached by a simple lattice quantization scheme that only quantizes the innovation, that is, the difference between the controller's belief about the current state and the true state. Via a separation principle between control and communication, similar results hold for causal lossy compression of additive noise Markov sources. Apart from standard dynamic programming arguments, our technical approach leverages the Shannon lower bound, develops new estimates for data compression with coding memory, and uses some recent results on high resolution variablelength vector quantization to prove that the new converse bounds are tight.
\end{abstract}

Index Terms-Linear stochastic control, LQR control, remote control, rate-distortion tradeoff, high resolution, causal ratedistortion theory, Gauss-Markov source.

\section{INTRODUCTION}

\section{A. System model}

Consider a discrete-time stochastic linear system:

$$
X_{i+1}=\mathrm{A} X_{i}+\mathrm{B} U_{i}+V_{i}
$$

where $X_{i} \in \mathbb{R}^{n}$ is the state, $V_{i} \in \mathbb{R}^{n}$ is the process noise, $U_{i} \in \mathbb{R}^{m}$ is the control action, and $\mathrm{A}$ and $\mathrm{B}$ are fixed matrices of dimensions $n \times n$ and $n \times m$, respectively. At time $i$, the controller observes output $G_{i}$ of the channel, and chooses a control action $U_{i}$ based on the data it has observed up to time $i$. At time $i$, the encoder observes the output of the sensor $Y_{i} \in \mathbb{R}^{k}$ :

$$
Y_{i}=\mathrm{C} X_{i}+W_{i}
$$

The authors are with California Institute of Technology (e-mail vkostina@caltech.edu, hassibi@caltech.edu).

A part of this work was presented at the 54th Annual Allerton Conference on Communication, Control and Computing [1].

The work of Victoria Kostina was supported in part by the National Science Foundation (NSF) under grants CCF-1566567 and CCF-1751356. The work of Babak Hassibi was supported in part by the NSF under grants CNS-0932428, CCF-1018927, CCF-1423663 and CCF-1409204, by a grant from Qualcomm Inc., by NASA's Jet Propulsion Laboratory through the President and Director's Fund, and by King Abdullah University of Science and Technology. where $C$ is a $k \times n$ deterministic matrix, and $W_{i} \in \mathbb{R}^{k}$ is the observation noise. The encoder forms a codeword $F_{i}$, which is then passed through the channel. Like the controller, the encoder has access to the entire history of the data it has observed. See Fig. 1.

We assume that system noises $V_{1}, V_{2} \ldots$ are i.i.d. zeromean, that observation noises $W_{1}, W_{2}, \ldots$ are zero-mean, i.i.d. independent of $\left\{W_{i}\right\}_{i=1}^{\infty}$, and that $X_{1}$ is zero-mean and independent of $\left\{V_{i}, W_{i}\right\}_{i=1}^{\infty}$. We make the usual assumption that the pair $(A, B)$ is controllable and that the pair $(A, C)$ is observable. If the encoder observes the full system state, i.e. $Y_{i}=X_{i}$ (rather than its noise-corrupted version as in (2)), then we say that the system is fully observed (rather than partially observed).

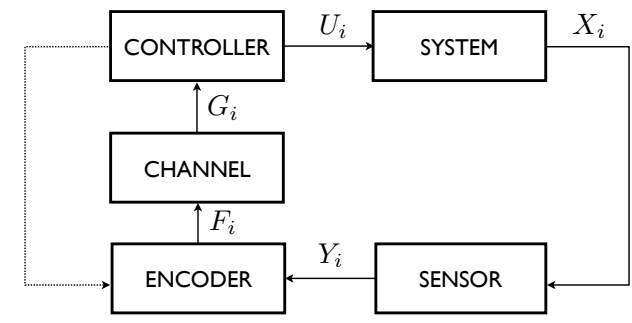

Fig. 1: The distributed control system.

Notation: Capital letters $X, Y$ denote (spatial) random vectors; $X^{t} \triangleq\left(X_{1}, \ldots, X_{t}\right)$ denotes temporal random vectors, or the history of vector samples up to time $t ; X_{i}^{t} \triangleq\left(X_{i}, \ldots, X_{t}\right)$ (empty if $t<i) ; X^{\infty} \triangleq\left(X_{1}, X_{2}, \ldots\right)$; for i.i.d. random vectors, $X$ denotes a random vector distributed the same as each of $X_{1}, X_{2}, \ldots ; \mathcal{D}$ represents a delay by one, i.e. $\mathcal{D} X^{t} \triangleq\left(0, X^{t-1}\right) ; \Sigma_{X} \triangleq \mathbb{E}\left[(X-\mathbb{E}[X])(X-\mathbb{E}[X])^{T}\right]$ denotes the covariance matrix of random vector $X ; X \Perp Y$ reads " $X$ is independent of $Y$ ". Sans-serif capitals A, B denote constant matrices; $A \succeq B(\succ B)$ signifies that $A-B$ is positive semidefinite (definite); $\mathrm{I}_{n}$ is the $n \times n$ identity matrix; $0_{k \times n}$ is the $k \times n$ all-zero matrix. Lowercase letters $a, b, \ldots$ denote known scalars. $\|\cdot\|$ denotes the Euclidean norm, $\triangleq$ reads "by definition"; $|\cdot|_{+} \triangleq \max \{0, \cdot\}$.

\section{B. The rate-cost tradeoff}

The efficiency of a given control law at time $t$ is measured by the linear quadratic regulator (LQR) cost function: ${ }^{1}$

$$
\operatorname{LQR}\left(X^{t}, U^{t-1}\right) \triangleq \mathbb{E}\left[\sum_{i=1}^{t-1}\left(X_{i}^{T} \mathrm{Q} X_{i}+U_{i}^{T} \mathrm{R} U_{i}\right)+X_{t}^{T} \mathrm{~S}_{t} X_{t}\right] \text {, }
$$

where $\mathrm{Q} \succeq 0, \mathrm{R} \succeq 0$ and $\mathrm{S}_{t} \succeq 0$. The LQR cost balances between the deviation of the system from the desired state $\mathbf{0}$ and the control power, which are defined with respect to the

\footnotetext{
${ }^{1}$ As common in information theory, here we abuse the notation slightly and write $\operatorname{LQR}\left(X^{t}, U^{t-1}\right)$ to mean that $\operatorname{LQR}()$ is a function of the joint distribution of $X^{t}, U^{t-1}$
} 
norms induced by the matrices $Q$ (and $S_{t}$ ) and $R$. In the special case $\mathrm{Q}=\mathrm{I}_{n}, \mathrm{R}=0$ and $\mathrm{S}_{t+1}=\mathrm{I}_{n}$, the cost function in (3) is the average mean-square deviation of the system from $\mathbf{0}$, $\mathbb{E}\left[\sum_{i=1}^{t}\left\|X_{i}\right\|^{2}\right]$.

Given a joint distribution of random vectors $U^{t}$ and $Y^{t}$, the directed mutual information is defined as [3]

$$
I\left(Y^{t} \rightarrow U^{t}\right) \triangleq \sum_{i=1}^{t} I\left(Y^{i} ; U_{i} \mid U^{i-1}\right) .
$$

Directed mutual information, which captures the information due to causal dependence of $U^{t}$ on $Y^{t}$, and which is less than or equal to the full mutual information $I\left(Y^{t} ; U^{t}\right)$, has proven useful in communication problems where causality and feedback play a role. Given a joint distribution $P_{Y^{t} U^{t}}$, it is enlightening to consider causally conditional probability kernel [4]

$$
P_{U^{t}|| Y^{t}} \triangleq \prod_{i=1}^{t} P_{U_{i} \mid U^{i-1}, Y^{i}}
$$

Note that $P_{Y^{t} U^{t}}=P_{Y^{t} \| \mathcal{D} U^{t}} P_{U^{t} \| Y^{t}}$. In Fig. 1, the system dynamics (1), (2) fixes the kernels $P_{Y^{t} \| \mathcal{D} U^{t}}, t=1,2, \ldots$, while the causal channels $P_{U^{t} \| Y^{t}}$ comprise the encoder, the channel and the controller.

The following information-theoretic quantity will play a central role in determining the operational fundamental limits of control under communication constraints.

Definition 1 (rate-cost function). The rate-cost function of the dynamical system $\left\{P_{Y^{t} \| \mathcal{D} U^{t}}\right\}_{t=0}^{\infty}$ is defined as

$$
\mathbb{R}(b) \triangleq \limsup _{t \rightarrow \infty} \inf _{\substack{P_{U^{t} \| Y^{t}}: \\ \frac{1}{t} \operatorname{LQR}\left(X^{t}, U^{t-1}\right) \leq b}} \frac{1}{t} I\left(Y^{t} \rightarrow U^{t}\right)
$$

In this paper, we will show a simple lower bound to the rate-cost function (6) of the stochastic linear system (1), (2). Although $\mathbb{R}(b)$ does not have a direct operational interpretation unless the channel is probabilistically matched [5] to the system, it is linked to the minimum data rate required to keep the system at LQR cost $b$, over both noiseless and noisy channels. Namely, we will show that $\mathbb{R}(b)$ provides a lower bound on the minimum capacity of the channel necessary to sustain LQR cost $b$, valid for any encoder/controller pair. We will also show that over noiseless channels, $\mathbb{R}(b)$ can be closely approached by a simple variable-length lattice-based quantization scheme that transmits only the innovation.

\section{Prior art}

The analysis of control under communication constraints has a rich history. The first results on the minimum data rate required for stabilizability appeared in [6], [7]. These works analyze the evolution of a scalar system from a worst-case perspective. In that setting, the initial state $X_{1}$ is assumed to belong to a bounded set, the process noise $V_{1}, V_{2}, \ldots$ is assumed to be bounded, and the system is said to be stabilizable if there exists a (rate-constrained) control sequence such that the worst-case deviation of the system state from the target state $\mathbf{0}$ is bounded: $\lim \sup _{t \rightarrow \infty}\left\|X_{t}\right\|<\infty$. In [6], [7], it was shown that a fully observed unstable scalar system can be kept bounded by quantized control if and only if the available data rate exceeds $\log \mathrm{A}$ bits per sample. Tatikonda and Mitter [8] generalized this result to vector systems; namely, they showed that the necessary data rate to stabilize a vector system with bounded noise is at least

$$
r>\sum_{i:\left|\lambda_{i}(\mathrm{~A})\right| \geq 1} \log \left|\lambda_{i}(\mathrm{~A})\right|,
$$

where the sum is over the unstable eigenvalues of $A$, i.e. those eigenvalues whose magnitude exceeds 1 . Compellingly, (7) shows that only the nonstable modes of A matter; the stable modes can be kept bounded at any arbitrarily small quantization rate (and even at zero rate if $V_{t} \equiv 0$ ). Using a volume-based argument, Nair et al. [9] showed a lower bound to quantization rate in order to attain $\limsup _{t \rightarrow \infty}\left\|X_{t}\right\| \leq d$, thereby refining (7). Nair et al. [9] also presented an achievability scheme confirming that for scalar systems, that bound is tight.

Nair and Evans [10] showed for systems with unbounded process and observation disturbances, Tatikonda and Mitter's condition on the rate (7) continues to be necessary and sufficient in order to keep the mean-square deviation of the plant state from 0 bounded, that is, in order to satisfy $\lim \sup _{t \rightarrow \infty} \mathbb{E}\left[\left\|X_{t}\right\|^{2}\right]<\infty$.

Nair and Evans' converse bound [10] applies to fixed-rate quantizers, that is, to compressors whose outputs can take one of $2^{r}$ values. Time-invariant fixed-rate quantizers are unable to attain bounded cost if the noise is unbounded [10], regardless of their rate. The reason is that since the noise is unbounded, over time, a large magnitude noise realization will inevitably be encountered, and the dynamic range of the quantizer will be exceeded by a large margin, not permitting recovery. Adaptive quantization schemes, which "zoom out" (i.e. stretch the quantization intervals) when the system is far from the target and "zoom in" when the system is close to the target, are studied in [10]-[13]. Structural properties of optimal zero-delay quantizers for the compression of Markov sources were investigated in [14]-[21].

In variable-rate (or length) quantization, the quantizer can have a countably infinite number of quantization cells. Entropy coding is applied to encode the indices of quantization cells, so that the more likely quantization cells have a shorter description and the less likely ones a longer one. Elia and Mitter [22] considered stabilization of a noiseless linear system controlled with a variable-length scalar quantizer, and showed that for a certain notion of coarseness, the coarsest quantizer has levels that follow a logarithmic law.

Beyond worst-case and mean-square stabilizability, Tatikonda et al. [2] considered a setting known as linear quadratic Gaussian (LQG) control ((1), (2) with Gaussian disturbances and LQR cost function in (3)) with communication constraints and tied the minimum attainable LQG cost to the Gaussian causal rate-distortion function, introduced decades earlier by Gorbunov and Pinsker [23], which is equal to the minimal (subject to a distortion constraint) directed mutual information between the stochastic process and its quantized representation [24]. Stabilizability of LQG systems under a directed mutual information constraint was studied in [25]. The problem of minimizing an arbitrary cost function in control of a general process under a directed mutual information constraint was formulated in [26]. Control 
of general Markov processes under a mutual information constraint was studied in [27]. Silva et al. [28] elucidated the operational meaning of directed mutual information, by pointing out that it lower-bounds the rate of a quantizer embedded into a feedback loop of a control system, and by showing that the bound is approached to within 1 bit by a dithered prefix-free quantizer, a compression setting in which both the compressor and the decompressor have access to a common dither - a random signal with special statistical properties. More recently, Silva et al. [29] computed a lower bound to the minimum quantization rate in scalar Gaussian systems with stationary disturbances and proposed a dithered quantization scheme that performs within 1.254 bits from it. Tanaka et al. [30] generalized the results of [29] to vector systems. A connection between causal rate-distortion function and Kalman filtering using dithered variable-length quantizers was explored in [31].

Causal rate-distortion function is challenging to evaluate, and beyond the scalar Gauss-Markov source [2], [23], no closed-form expression is known for it. For stationary scalar Gaussian processes, Derpich and Ostergaard [32] showed an upper bound and Silva et al. [29] a lower bound. For vector Gauss-Markov sources, Tanaka et al. developed a semidefinite program to compute exactly the minimum directed mutual information in quantization [33] and control [34].

\section{Our contribution}

In this paper, we show a lower bound to $\mathbb{R}(b)$ of a fully observed system. We do not require the noise $V_{i}$ to be bounded or Gaussian. We also show that (7) remains necessary to keep the LQR cost bounded, even if the system noise is nonGaussian, generalizing previously known results. Although our converse lower bound holds for a general class of codes that can take full advantage of the memory of the data observed so far and that are not constrained to be linear or have any other particular structure, we show that the new bound can be closely approached within a much more narrow class of codes. Namely, a simple variable-length quantization scheme, which uses a lattice covering and which only transmits the difference between the controller's estimate about the current system state and the true state, performs within a fraction of a bit from the lower bound, with a vanishing gap as $b$ approaches its minimum attainable value, $b_{\min }$. The scheme is a variant of a classical differential pulse-code modulation (DPCM) scheme, in which a variable-length lattice code is used to encode the innovation process. Unlike previously proposed quantization schemes with provable performance guarantees, our scheme does not use dither.

Our results generalize to partially observed systems, where the encoder does not have access to $X_{i}$ but only to its noisecorrupted version, $Y_{i}$. For those results to hold, we require the system and observation noises to be Gaussian.

Our approach is based on a new lower bound to causal ratedistortion function, termed the causal Shannon lower bound (Theorem 9 in Section III below), which holds for vector Markov sources with continuous additive disturbances, as in (1). For the scalar Gauss-Markov source, the bound coincides with a previously known expression [23].

\section{E. Technical approach}

The main idea behind our approach to show a converse (impossibility) result is to recursively lower-bound distortionrate functions arising at each step. We apply the classical Shannon lower bound [35], which bounds the distortion-rate function $X$ in terms of the entropy power of $X$, and we use the entropy power inequality [36], [37] to split up the distortionrate functions of sums of independent random variables. Since Shannon's lower bound applies regardless of the distribution of the source random variable, our technique circumvents a precise characterization of the distribution of the state at each time instant. The technique also does not restrict the system noises to be Gaussian.

To show that our bound can be approached at high rates, we build on the ideas from high resolution quantization theory. A pioneering result of Gish and Piece [38] states that in the limit of high resolution, a uniform scalar quantizer incurs a loss of only about $\frac{1}{2} \log _{2} \frac{2 \pi e}{12} \approx 0.254$ bits per sample. Ziv [39] showed that regardless of target distortion, the normalized output entropy of a dithered scalar quantizer exceeds that of the optimal vector quantizer by at most $\frac{1}{2} \log \frac{4 \pi e}{12} \approx 0.754$ bits per sample. A lattice quantizer presents a natural extension of a scalar uniform quantizer to multiple dimensions. The advantage of lattice quantizers over uniform scalar quantizers is that the shape of their quantization cells can be made to approach a Euclidean ball in high dimensions [40]. Furthermore, the entropy rate of dithered lattice quantizers converges to Shannon's lower bound in the limit of vanishing distortion [41]-[43].

While the presence of a dither signal both at the encoder and the decoder greatly simplifies the analysis and can improve the quantization performance, it also complicates the engineering implementation. In this paper, we do not consider dithered quantization. Neither do we rely directly on the classical heuristic reasoning by Gish and Piece [38]. Instead, we use a non-dithered lattice quantizer followed by an entropy coder. To rigorously prove that its performance approaches our converse bound, we employ a recent upper bound [44] on the output entropy of lattice quantizers in terms of the differential entropy of the source, the target distortion and a smoothness parameter of the source density.

\section{F. Paper organization}

In Section II, we state and discuss our main results: Section II-A focuses on the scenario where the observer sees the system state (fully observed system), Section II-B discusses a generalization to the scenario where the observer sees a noisy measurement of the system state (partially observed system), and Section II-C discusses the operational implications of our bounds in the settings of fixed-rate quantization, variablerate quantization and joint source-channel coding. In Section III, we introduce the causal lossy compression problem, and we state the causal Shannon lower bound, together with a matching achievability result. In Section IV, we discuss separation between control, estimation and communication, a structural result that allows us to disentangle the three tasks. The proofs of the converse results are given in Section V, and the achievability schemes are presented in Section VI. 


\section{MAIN RESULTS}

\section{A. Fully observed system}

In the absence of communication constraints, the minimum LQR cost attainable in the limit of infinite time is:

$$
b_{\min }=\operatorname{tr}\left(\Sigma_{V} S\right)
$$

where $\Sigma_{V}$ is the covariance matrix of each of the $V_{1}, V_{2}, \ldots$, and $S$ is the solution to the algebraic Riccati equation

$$
\begin{aligned}
& \mathrm{S}=\mathrm{Q}+\mathrm{A}^{T}(\mathrm{~S}-\mathrm{M}) \mathrm{A} \\
& \mathrm{M} \triangleq \mathrm{L}^{T}\left(\mathrm{R}+\mathrm{B}^{T} \mathrm{SB}\right) \mathrm{L}=\mathrm{SB}\left(\mathrm{R}+\mathrm{B}^{T} \mathrm{SB}\right)^{-1} \mathrm{~B}^{T} \mathrm{~S} \\
& \mathrm{~L} \triangleq\left(\mathrm{R}+\mathrm{B}^{T} \mathrm{SB}\right)^{-1} \mathrm{~B}^{T} \mathrm{~S} .
\end{aligned}
$$

Our results quantifying the overhead over (8) due to communication constraints are expressed in terms of the entropy power of the system and observation noises. The entropy power of an $n$-dimensional random vector $X$ is ${ }^{2}$

$$
N(X) \triangleq \frac{1}{2 \pi e} \exp \left(\frac{2}{n} h(X)\right) \text {, }
$$

where $h(X)=-\int_{\mathbb{R}^{n}} f_{X}(x) \log f_{X}(x) d x$ is the differential entropy of $X$, and $f_{X}(\cdot)$ is the density of $X$ with respect to the Lebesgue measure on $\mathbb{R}^{n}$. The entropy power satisfies the following classical inequalities:

$$
N(X) \leq\left(\operatorname{det} \Sigma_{X}\right)^{\frac{1}{n}} \leq \frac{1}{n} \operatorname{Var}[X] .
$$

The first equality in (99) is attained if and only if $X$ is Gaussian and the second if and only if $X$ is white.

Our first result is a lower bound on the rate-cost function.

Theorem 1. Consider the fully observed linear stochastic system (1). Suppose that $h(V)>-\infty$. At any LQR cost $b>\operatorname{tr}\left(\Sigma_{V} S\right)$, the rate-cost function is bounded below as

$$
\mathbb{R}(b) \geq \log |\operatorname{det} \mathrm{A}|+\frac{n}{2} \log \left(1+\frac{N(V)|\operatorname{det} \mathrm{M}|^{\frac{1}{n}}}{\left(b-\operatorname{tr}\left(\Sigma_{V} \mathrm{~S}\right)\right) / n}\right),
$$

where $\mathrm{M}$ is defined in (10).

The bound in Theorem 1 is nontrivial if $M \succ 0$, which happens if rank $\mathrm{B}=n$ and either $\mathrm{Q} \succ 0$ or $\mathrm{R} \succ 0$. The bound in Theorem 1 continues to hold whether or not at time $i$ the encoder observes the previous control inputs $U_{1}, U_{2}, \ldots, U_{i-1}$.

The right-hand side of (14) is a decreasing function of $b$, which means that the controller needs to know more information about the state of the system to attain a smaller target cost. As an important special case, consider the ratecost tradeoff where the goal is to minimize the mean-square deviation from the desired state $\mathbf{0}$. Then, $\mathrm{Q}=\mathrm{I}_{n}, \mathrm{R}=0$, $\mathrm{S}=\mathrm{M}=\mathrm{I}_{n}, b_{\min }=\operatorname{Var}[V]$, and (14) particularizes as

$$
\mathbb{R}(b) \geq \log |\operatorname{det} \mathrm{A}|+\frac{n}{2} \log \left(1+\frac{N(V)}{(b-\operatorname{Var}[V]) / n}\right) .
$$

In another important special case, namely Gaussian $V$, (14) particularizes as

$$
\mathbb{R}(b) \geq \log |\operatorname{det} \mathrm{A}|+\frac{n}{2} \log \left(1+\frac{\left|\operatorname{det} \Sigma_{V} \mathrm{M}\right| \frac{1}{n}}{\left(b-\operatorname{tr}\left(\Sigma_{V} \mathrm{~S}\right)\right) / n}\right)
$$

\footnotetext{
${ }^{2}$ All log's and exp's are common arbitrary base specifying the information units.
}

For the scalar Gaussian system, (16) holds with equality. This is a consequence of known analyses [23], [2], [32, Th. 3] (see also Remarks 5 and 6 in Section III below).

A typical behavior of (15) is plotted in Fig. 3 as a function of target cost $b$. As $b \downarrow b_{\min }$, the required rate $\mathbb{R}(b) \uparrow \infty$. Conversely, as $b \uparrow \infty$, the rate monotonically decreases and approaches $\log |\operatorname{det} A|$. The rate-cost tradeoff provided by Theorem 1 can serve as a gauge for choosing an appropriate communication rate in order to meet the control objective. For example, in the setting of Fig. 3, decreasing the data rate below 1 nat per sample incurs a massive penalty in cost, because the bound is almost flat in that regime. On the other hand, increasing the rate from 1 to 3 nats per sample brings a lot of improvement in cost, while further increasing it beyond 3 nats results in virtually no improvement.

Also plotted in Fig. 3 is the output entropy of a variable-rate uniform scalar quantizer that takes advantage of the memory of the past only through the innovation, i.e. the difference between the controller's prediction of the state at time $i$ given the information the controller had at time $i-1$ and the true state (see Section VI for a precise description of the quantizer) Its performance is strikingly close to the lower bound, being within 0.5 nat even at large $b$, despite the fact that quantizers in this class cannot attain the optimal cost exactly [45]. The gap further vanishes as $b$ decreases. The gap can be further decreased for multidimensional systems by taking advantage of lattice quantization. These effects are formally captured by the achievability result we are about to present, Theorem 2.

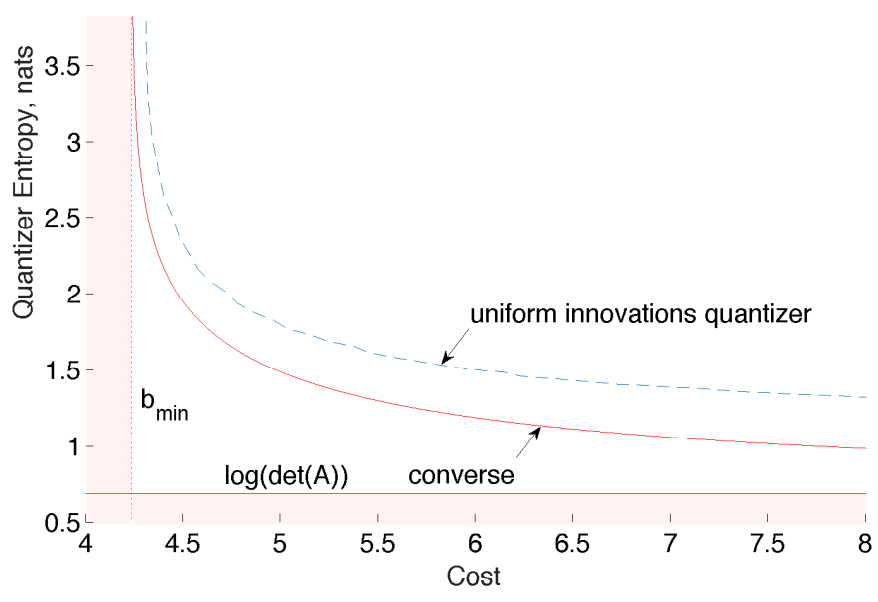

Fig. 2: The minimum quantizer entropy compatible with cost $b$ in a fully observed system (1) with parameters $n=1, \mathrm{~A}=2, \mathrm{~B}=\mathrm{Q}=$ $\mathrm{R}=1, V$ has Laplace distribution with variance 1 .

Replacing in Definition 1 the directed mutual information by the entropy of a causal quantizer, we introduce

Definition 2 (entropy-cost function). The entropy-cost function of the dynamical system $\left\{P_{Y^{t}} \| \mathcal{D} U^{t}\right\}_{t=0}^{\infty}$ is defined as

$$
\mathbb{H}(b) \triangleq \limsup _{t \rightarrow \infty} \inf _{\substack{P_{U^{t} \| Y^{t}}: \\ \frac{1}{t} \mathrm{LQR}\left(X^{t}, U^{t-1}\right) \leq b}} H\left(U^{t}\right) .
$$

Since $I\left(Y^{i} ; U_{i} \mid U^{i-1}\right) \leq H\left(U_{i} \mid U^{i-1}\right)$, we have

$$
\mathbb{H}(b) \geq \mathbb{R}(b) .
$$


On the other hand, there exists a variable-length quantizer that keeps the system at cost $b$ and whose average encoded length does not exceed $\mathbb{H}(b)$ (see Section II-C for details). Thus, unlike $\mathbb{R}(b)$, the function $\mathbb{H}(b)$ has a direct operational interpretation.

Theorem 2, presented next, holds under the assumption that the density of the noise is sufficiently smooth. Specifically, we adopt the following notion of a regular density.

Definition 3 (Regular density, [46]). Let $c_{0} \geq 0, c_{1} \geq 0$. Differentiable probability density function $f_{X}$ of a random vector $X \in \mathbb{R}^{n}$ is called $\left(c_{0}, c_{1}\right)$-regular if ${ }^{3}$

$$
\left\|\nabla f_{X}(x)\right\| \leq\left(c_{1}\|x\|+c_{0}\right) f_{X}(x), \quad \forall x \in \mathbb{R}^{n} .
$$

A wide class of densities satisfying smoothness condition (19) is identified in [46]. Gaussian, exponential, uniform Gamma, Cauchy, Pareto distributions are all regular. Convolution with Gaussians produces a regular density: more precisely, the density of $B+Z$, with $B \Perp Z$ and $Z \sim \mathcal{N}\left(0, \sigma^{2} \mathrm{I}\right)$, is $\left(\frac{4}{\sigma^{2}} \mathbb{E}[\|B\|], \frac{3}{\sigma^{2}}\right)$-regular. Likewise, if the density of $Z$ is $\left(c_{0}, c_{1}\right)$-regular, then that of $B+Z$, where $\|B\| \leq b$ a.s., $B \Perp Z$ is $\left(c_{0}+c_{1} b, c_{1}\right)$-regular.

Theorem 2. Consider the fully observed linear stochastic system (1), $Y_{i}=X_{i}$. Suppose that $\mathrm{M} \succ 0$ and that $V$ has a regular density. Then, at any $L Q R$ cost $\left.b>\operatorname{tr}\left(\Sigma_{V} S\right)\right)$, the entropy-cost function is bounded by

$$
\begin{aligned}
\mathbb{H}(b) & \leq \log |\operatorname{det} \mathrm{A}|+\frac{n}{2} \log \left(1+\frac{N(V)|\operatorname{det} \mathrm{M}|^{\frac{1}{n}}}{\left(b-\operatorname{tr}\left(\Sigma_{V} \mathrm{~S}\right)\right) / n}\right) \\
& +O_{1}(\log n)+O_{2}\left(\left(b-\operatorname{tr}\left(\Sigma_{V} \mathrm{~S}\right)\right)^{\frac{1}{2}}\right),
\end{aligned}
$$

where $O_{1}(\log n) \leq C_{1} \log n$ and $O_{2}(\xi) \leq C_{2} \min \left\{\xi, c_{2}\right\}$ for some nonnegative constants $C_{1}, C_{2}$ and $c_{2}$.

The first two terms in (20) match the first two terms in (14). The $O_{1}(\log n)$ term is the penalty due to the shape of lattice quantizer cells not being exactly spherical, i.e. it is the penalty due to the space-filling loss of the quantizer at finite $n$. In Section VI, we provide a precise expression for that term for $n=1,2, \ldots$. The $O_{2}\left(\left(b-\operatorname{tr}\left(\Sigma_{V} S\right)\right)^{\frac{1}{2}}\right)$ is the penalty due to the distribution of the innovation not being uniform. It becomes negligible for small $b-\operatorname{tr}\left(\Sigma_{V} S\right)$, and the speed of that convergence depends on the smoothness parameters of the noise density.

Theorem 2 implies that if the channel $F_{i} \rightarrow G_{i}$ is noiseless, then there exists a quantizer with output entropy given by the right side of (20) that attains LQR cost $b>\operatorname{tr}\left(\Sigma_{V} S\right)$ ), when coupled with an appropriate controller. In fact, the bound in (20) is attainable by a simple lattice quantization scheme that only transmits the innovation of the state (a DPCM scheme). The controller computes the control action based on the quantized data as if it was the true state (the so-called certainty equivalence control).

Theorem 1 gives a lower (converse) bound on the output entropy of quantizers that achieve the target cost $b$, without making any assumptions on the quantizer structure and permitting the use of the entire history of observation data.

\footnotetext{
${ }^{3}$ As usual, $\nabla$ denotes the gradient.
}

Theorem 2 proves that the converse can be approached by a strikingly simple quantizer coupled with a standard controller, without common randomness (dither) at the encoder and the decoder. Furthermore, although nonuniform rate allocation across time is allowed by Definition 2, such freedom is not needed to achieve (20); the scheme that achieves (20) satisfies $H\left(U_{i} \mid U^{i-1}\right) \rightarrow r$ in the limit of large $i$.

Although the bound in Theorem 1 is tight at low $b$ (as demonstrated by Theorem 2), if A has both stable and unstable eigenvalues and $b$ is large, it is possible to improve the bound in Theorem 1 by projecting out the stable modes of the system. Towards this end, consider a Jordan decomposition of A: ${ }^{4}$

$$
\mathrm{A}=\mathrm{JA}^{\prime} \mathrm{J}^{-1} \text {, }
$$

where $A^{\prime}$ is the Jordan form of $A$, and $J$ is invertible. Without loss of generality, assume the eigenvalues of $A^{\prime}$ are ordered in decreasing magnitude order. Write $A^{\prime}$ as a direct sum of its Jordan blocks, $\mathrm{A}^{\prime}=\mathrm{A}_{1} \oplus \ldots \oplus \mathrm{A}_{\bar{s}}$. For some $s$ such that $1 \leq$ $s \leq \bar{s} \leq n$, let $\ell=\operatorname{dim}\left(\mathrm{A}_{1}\right)+\ldots+\operatorname{dim}\left(\mathrm{A}_{s}\right)$ be the dimension of the column space of the first $s$ Jordan blocks. Consider the orthogonal projection matrix onto that space, $\Pi_{\ell} \Pi_{\ell}^{T}$, where $\Pi_{\ell}$ is a 0 -1-valued $n \times \ell$ matrix given by:

$$
\Pi_{\ell} \triangleq\left[\begin{array}{c}
I_{\ell} \\
0
\end{array}\right]
$$

The improvement of Theorem 1 can now be formulated.

Theorem 3. Consider the fully observed linear stochastic system (1), $Y_{i}=X_{i}$. Suppose that $h(V)>-\infty$. Let $\Lambda$ be a diagonal matrix such that $\mathrm{M}^{\prime} \triangleq \mathrm{J}^{T} \mathrm{MJ} \succeq \Lambda$, where $\mathrm{J}$ is defined in (21). At any $L Q R$ cost $b>\operatorname{tr}\left(\Sigma_{V} S\right)$, the rate-cost function is bounded below as

$$
\mathbb{R}(b) \geq \ell \log a^{\prime}+\frac{\ell}{2} \log \left(1+\frac{\mu^{\prime} N\left(\Pi_{\ell}^{T} \mathrm{~J}^{-1} V\right)}{\left(b-\operatorname{tr}\left(\Sigma_{V} \mathrm{~S}\right)\right) / \ell}\right) .
$$

where $\Pi_{\ell}$ is defined in (22), and

$$
\begin{aligned}
& a^{\prime} \triangleq\left|\operatorname{det}\left(\Pi_{\ell}^{T} \mathrm{~J}^{-1} \mathrm{AJ} \Pi_{\ell}\right)\right|^{\frac{1}{\ell}}, \\
& \mu^{\prime} \triangleq(\operatorname{det} \Lambda)^{\frac{1}{\ell}} .
\end{aligned}
$$

If $\ell=n$, then $a^{\prime}=|\operatorname{det} \mathrm{A}|$, and the bound in (23) reduces to (14). On the other hand, taking $\ell$ to be the number of unstable eigenvalues in (23), one can conclude

$$
\mathbb{R}(b) \geq \sum_{i:\left|\lambda_{i}(\mathrm{~A})\right| \geq 1} \log \left|\lambda_{i}(\mathrm{~A})\right| .
$$

If the dimensionality of control is less than that of the system, $m<n$, the bounds in Theorem 1 and Theorem 3 reduce to $\log |\operatorname{det} \mathrm{A}|$ and $\ell \log a^{\prime}$, respectively, losing the dependence on $b$. The bound in Theorem 4 below is a decreasing function of $b$, even if $m<n$.

Theorem 4. Consider the fully observed linear stochastic system (1), $Y_{i}=X_{i}$. Suppose that $h(V)>-\infty$. At any $L Q R$ cost $b>\operatorname{tr}\left(\Sigma_{V} S\right)$, the rate-cost function satisfies

$$
\begin{aligned}
& \mathbb{R}(b) \geq \log |\operatorname{det} \mathrm{A}|+\frac{m}{2} \log \left(\frac{a^{2}}{|\operatorname{det} \mathrm{A}|^{2}}+\frac{\mu N(V)^{\frac{n}{m}} m}{b-\operatorname{tr}\left(\Sigma_{V} \mathrm{~S}\right)}\right), \\
& \text { where } \quad a \triangleq \inf _{i \geq 1}\left(\frac{\operatorname{det}\left(\mathrm{LA}^{i} \Sigma_{V} \mathrm{~A}^{i T} \mathrm{~L}^{T}\right)}{\operatorname{det} \Sigma_{V} \operatorname{det}\left(\mathrm{LL}^{T}\right)}\right)^{\frac{1}{2 i m}}
\end{aligned}
$$

\footnotetext{
${ }^{4}$ A Jordan decomposition of A has been previously applied in the context of control under communication constraints in e.g. [8], [10].
} 


$$
\mu \triangleq\left(\operatorname{det}\left(\mathrm{R}+\mathrm{B}^{T} \mathrm{SB}\right) \operatorname{det}\left(\mathrm{LL}^{T}\right)\right)^{\frac{1}{m}},
$$

and $\mathrm{L}$ is defined in (11).

If $m=n$, Theorem 4 reduces to Theorem 1 .

We conclude Section II-A with a few technical remarks.

Remark 1. Since the running mean-square cost is bounded above as $\frac{1}{t} \mathbb{E}\left[\sum_{i=1}^{t}\left\|X_{i}\right\|^{2}\right] \leq \max _{1 \leq i \leq t} \mathbb{E}\left[\left\|X_{i}\right\|^{2}\right]$, our Theorem 3 implies (via (26)) the weaker result of Nair and Evans [10], who showed the necessity of (26) to keep $\sup _{t} \mathbb{E}\left[\left\|X_{t}\right\|^{2}\right]$ bounded. Note also that the approach of Nair and Evans [10] applies only to fixed-rate quantization, while our approach encompasses both fixed- and variable-rate quantization, as well as control over noisy channels and non-Gaussian system disturbances.

Remark 2. Tatikonda et al. [2, (17)] proposed to apply the classical reverse waterfilling, known to achieve the noncausal Gaussian rate-distortion function, at each step $i$, to compute the causal Gaussian rate-distortion function for the sequence of vectors $\left\{X_{i}\right\}_{i=1}^{\infty}$. Unfortunately, reverse waterfilling is only suboptimal, as can be verified numerically by comparing [2, (17)] to the semidefinite program of Tanaka et al. [33] (Fig. 3). The reason reverse waterfilling does not apply is that $\lambda_{i}(t)$ in $[2,(15)]$ depend on the distortion threshold, an effect not present in the computation of the classical non-causal ratedistortion function.

Remark 3. The semidefinite program (SDP) of Tanaka et al. [33] provides an exact numerical solution to the Gaussian causal rate-distortion-function, while our results in Theorems 1, 3, 4 provide analytical lower bounds, which hold beyond Gaussian noise. Fig. 3 presents a numerical comparison between our lower bound in Theorem 3 and the exact calculation of $\mathbb{R}(d)$, for a randomly generated 3-dimensional system. For the example in Fig. 3, our lower bound is within 0.14 bits from the optimum. While always tight in low-cost regime, in medium-cost regime it will become looser if the spread of the eigenvalues of $A$ is large.

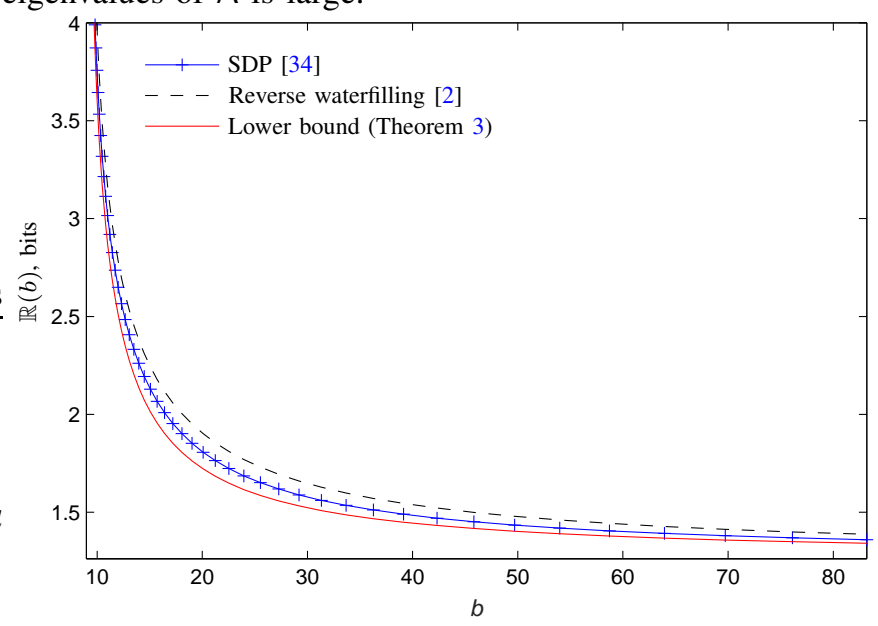

Fig. 3: Our lower bound in Theorem 3, the exact rate-cost function computed using the SDP method [34], and the reverse watefilling solution [2], computed for a Gaussian $V, \mathrm{~A}=\operatorname{diag}(2,1.2, .3), \mathrm{Q}=$ $\mathrm{R}=\mathrm{I}$; B and $\Sigma_{V}$ were generated randomly. The reverse watefilling solution is evidently strictly suboptimal.

\section{B. Partially observed system}

Consider now the scenario in which the encoder sees a noisy observation of the system state and forms a codeword to transmit to the controller using its present and past noisy observations. If the system and observation noises are jointly Gaussian, our results in Section II-A generalize readily.

In the absence of communication constraints, the minimum cost decomposes into two terms, the cost due to the noise in the system in (8), and the cost due to the noise in the observation of the state:

$$
b_{\text {min }}=\operatorname{tr}\left(\Sigma_{V} \mathrm{~S}\right)+\operatorname{tr}\left(\Sigma \mathrm{A}^{T} \mathrm{MA}\right),
$$

where $\Sigma$ is the covariance matrix of the estimation error $X_{t}-\mathbb{E}\left[X_{t} \mid Y^{t}, U^{t-1}\right]$ in the limit $t \rightarrow \infty$. The celebrated separation principle of estimation and control states that the minimum cost in (30) can be attained by separately estimating the value of $X_{i}$ using the noisy observations $Y^{i}$, and by applying the optimal control to the estimate as if it was the true state of the system. If system and observation noises are Gaussian, the optimal estimator admits a particularly elegant implementation via the Kalman filter. Then, $\Sigma$ is given by

$$
\Sigma=\mathrm{P}-\mathrm{K}\left(\mathrm{CPC}^{T}+\Sigma_{W}\right) \mathrm{K}^{T},
$$

where $\Sigma_{W}$ is the covariance matrix of each $W_{i}, \mathrm{P}$ is the solution to the algebraic Riccati equation

$$
\mathrm{P}=\mathrm{APA}^{T}-\mathrm{AK}\left(\mathrm{CPC}^{T}+\Sigma_{W}\right) \mathrm{K}^{T} \mathrm{~A}^{T}+\Sigma_{V},
$$

and $\mathrm{K}$ is the steady state Kalman filter gain:

$$
\mathrm{K} \triangleq \mathrm{PC}^{T}\left(\mathrm{CPC}^{T}+\Sigma_{W}\right)^{-1} \text {. }
$$

As Theorem 5 below shows, the rate-cost function is bounded in terms of the steady state covariance matrix of the innovation in encoder's state estimate:

$$
\mathrm{N} \triangleq \mathrm{K}\left(\mathrm{CPC}^{T}+\Sigma_{W}\right) \mathrm{K}^{T}=\mathrm{A} \Sigma \mathrm{A}^{T}-\Sigma+\Sigma_{V} .
$$

Theorem 5. Consider the partially observed linear stochastic system (1), (2). Suppose further that $X_{1}, V$ and $W$ are all Gaussian. At any target $L Q R$ cost $b>b_{\min }$, the rate-cost function is bounded below as

$$
\mathbb{R}(b) \geq \log |\operatorname{det} \mathrm{A}|+\frac{n}{2} \log \left(1+\frac{(\operatorname{det} \mathrm{NM})^{\frac{1}{n}}}{\left(b-b_{\min }\right) / n}\right) .
$$

The bound in Theorem 5 is nontrivial if both $\mathrm{M} \succ 0$ and $\mathrm{N} \succ 0$. The necessary condition for that is $\operatorname{rank} \mathrm{B}=$ $\operatorname{rank} \mathrm{C}=n$. In the fully observed system, $\mathrm{C}=\mathrm{K}=\mathrm{I}$, $\mathrm{P}=\mathrm{N}=\Sigma_{V}, \Sigma_{W}=0$, and (35) reduces to (16). As we will see in Section $\mathrm{V}$, the key to proving Theorem 5 is to represent the evolution of the current encoder's estimate of the state in terms of its previous best estimate and an independent Gaussian, as carried out by the Kalman filter recursion. It is for this technique to apply that we require the noises to be Gaussian. ${ }^{5}$ As for the achievable scheme, as in the fully observed case, the observer quantizes the innovation, only now the innovation is the difference between the observer's estimate and the controller's estimate. The scheme operates as follows. The observer sees $Y_{i}$, recursively computes its estimate of the state $X_{i}$, computes the innovation, quantizes the innovation. The controller receives the quantized value

${ }^{5}$ Recall that Theorems 1, 2, 3 make no such restrictions. 
of the innovation, recursively computes its own estimate of the state $X_{i}$, and forms the control action that is a function of controller's state estimate only. Accordingly, the tasks of state estimation, quantization and control are separated. The momentous insight afforded by Theorem 6 below is that in the high rate regime, this simple scheme performs provably close to $\mathbb{R}(b)$, the best rate-cost tradeoff theoretically attainable.

Theorem 6. Consider the partially observed linear stochastic system (1), (2). Suppose that $\mathrm{M} \succ 0, \mathrm{~N} \succ 0$, and that $X_{1}, V$ and $W$ are all Gaussian. At any LQR cost $b>b_{\min }$, the entropy-cost function is bounded above as

$$
\begin{aligned}
\mathbb{H}(b) & \leq \log |\operatorname{det} \mathrm{A}|+\frac{n}{2} \log \left(1+\frac{|\operatorname{det} \mathrm{NM}|^{\frac{1}{n}}}{\left(b-b_{\min }\right) / n}\right) \\
& +O_{1}(\log n)+O_{2}\left(\left(b-b_{\text {min }}\right)^{\frac{1}{2}}\right),
\end{aligned}
$$

where $O_{1}(\log n) \leq C_{1} \log n$ and $O_{2}(\xi) \leq C_{2} \min \left\{\xi, c_{2}\right\}$ for some nonnegative constants $C_{1}, C_{2}$ and $c_{2}$.

Theorems 3 and 4 generalize as follows.

Theorem 7. Consider the partially observed linear stochastic system (1), (2). Suppose that (A, B) is controllable and (A, C) is observable. Suppose further that $X_{1}, V$ and $W$ are all Gaussian. At any $L Q R$ cost $b>b_{\min }$, the rate-cost function is bounded from below as

$$
\mathbb{R}(b) \geq \log a^{\prime}+\frac{1}{2} \log \left(1+\frac{\eta^{\prime} \mu^{\prime}}{\left(b-b_{\min }\right) / \ell}\right) .
$$

where $a^{\prime}, \mu^{\prime}$ are defined in (24)-(25), and

$$
\eta^{\prime} \triangleq\left(\operatorname{det}\left(\Pi_{\ell}^{T} \mathrm{~J}^{-1} \mathrm{NJ}^{-1 T} \Pi_{\ell}\right)\right)^{\frac{1}{\ell}},
$$

where $\mathrm{J}$ and $\Pi_{\ell}$ are defined in (21), (22), respectively.

Theorem 8. Consider the partially observed linear stochastic system (1), (2). Suppose that (A, B) is controllable, (A, C) is observable, and that $m \leq k \leq n$. Suppose further that $X_{1}, V$ and $W$ are all Gaussian. At any LQR cost $b>b_{\min }$, the rate-cost function is bounded from below as

$\mathbb{R}(b) \geq \log |\operatorname{det} \mathrm{A}|+\frac{m}{2} \log \left(\frac{a^{2}}{|\operatorname{det} \mathrm{A}|^{2}}+\frac{\eta \mu}{\left(b-b_{\min }\right) / m}\right)$,

where $\mu$ is defined in (29),

$$
\begin{aligned}
& a \triangleq \inf _{i \geq 1}\left(\frac{\operatorname{det}\left(\mathrm{LA}^{i} \mathrm{~K}\left(\mathrm{CPC}^{T}+\Sigma_{W}\right) \mathrm{K}^{T} \mathrm{~A}^{i T} \mathrm{~L}^{T}\right)}{\operatorname{det}\left(\mathrm{CPC}^{T}+\Sigma_{W}\right) \operatorname{det}\left(\mathrm{LL}^{T}\right) \operatorname{det}\left(\mathrm{K}^{T} \mathrm{~K}\right)}\right)^{\frac{1}{2 i m}}, \\
& \eta \triangleq\left(\operatorname{det}\left(\mathrm{CPC}^{T}+\Sigma_{W}\right) \operatorname{det}\left(\mathrm{K}^{T} \mathrm{~K}\right)\right)^{\frac{1}{m}}
\end{aligned}
$$

and $\mathrm{L}, \mathrm{P}, \mathrm{K}$ are defined in (11), (32), (33), respectively.

If $k=m=n$, Theorem 8 reduces to Theorem 5 .

\section{Operational implications}

So far we have formally defined the rate-cost / entropycost functions as the limiting solutions of a minimal directed mutual information / entropy subject to an LQR cost constraint (Definition 1 / Definition 2) and presented lower and upper bounds to those functions. In this section we discuss the operational implications of the results in Section II-A and Section II-B in several communication scenarios.

\section{1) Control over a noisy channel}

Consider first a general scenario in which the channel in Fig. 1 is a dynamical channel defined by causal kernels $\left\{P_{G^{t} \| F^{t}}\right\}_{t=1}^{\infty}$. For the class of directed information stable channels, the feedback capacity of the channel is [47], [48]

$$
C=\liminf _{t \rightarrow \infty} \sup _{P_{F^{t} \| \mathcal{D} G^{t}}} \frac{1}{t} I\left(F^{t} \rightarrow G^{t}\right),
$$

If past channel outputs are not available at the encoder, then the sup is over all $P_{F^{t}}$, and $I\left(F^{t} \rightarrow G^{t}\right)=I\left(F^{t} ; G^{t}\right)$.

The following result, the proof of which is deferred until Section IV, implies that the converse results in Theorems 1, 3, 5 and 7 present lower bounds on the capacity of the channel necessary to attain $b$.

Proposition 1. A necessary condition for stabilizing the system in (1), (2) at LQR cost b is,

$$
\mathbb{R}(b) \leq C .
$$

One remarkable special case when equality in (42) is attained is control of a scalar Gaussian system over a scalar memoryless AWGN channel [2], [49]-[51]. In that case, the channel is probabilistically matched to the data to be transmitted [5], no coding beyond simple amplification is needed, and linearly transmitting the innovation is optimal [49]. In practice, such matching rarely occurs, and intelligent joint source-channel coding techniques can lead to a significant performance improvement. One such technique that approaches (42) in a particular scenario is discussed in [51]. In general, how closely the bound in (42) can be approached over noisy channels remains an open problem.

2) Control under fixed-rate quantization

If the channel connecting the encoder to the controller is a noiseless bit pipe that accepts a fixed number of $r$ bits per channel use (so that $C=r$ ), both $\mathbb{R}(b)$ and $\mathbb{H}(b)$ are lower bounds on the minimum quantization rate $r$ required to attain cost $b$ :

$$
\mathbb{R}(b) \leq \mathbb{H}(b) \leq r .
$$

Therefore, the converse results in Theorems 1, 3, 4, 5, 7 and 8 give sharp lower bounds on the minimum size of a fixedrate quantizer compatible with LQR $\operatorname{cost} b$. The achievability results in Theorems 2 and 6 are insufficient to establish the existence of a fixed-rate quantizer of a rate approaching $\mathbb{R}(b)$. While attempting to find a time-invariant fixed-rate quantizer operating at any finite cost is futile [10], determining whether there exists an adaptive fixed-rate quantization scheme approaching the converses in Theorems 1 and 5 remains an intriguing open question.

\section{3) Control under variable-length quantization}

Assume now that the channel connecting the encoder to the controller is a noiseless channel that accepts an average of $r$ bits per sample. That is, the channel input alphabet is the set of all binary strings, $\{\emptyset, 0,1,00,01, \ldots\}$, and the encoding function $P_{F^{t} \| Y^{t}}$ must be such that

$$
\frac{1}{t} \sum_{i=1}^{t} \mathbb{E}\left[\ell\left(F_{i}\right)\right] \leq r
$$

where $\ell\left(F_{i}\right)$ denotes the length of the binary string $F_{i}$. 
The minimum encoded average length $L^{\star}(X)$ in lossless compression of object $X$ is bounded as [52], [53]

$$
\begin{aligned}
L^{\star}(X) & \leq H(X) \\
& \leq L^{\star}(X)+\log _{2}\left(L^{\star}(X)+1\right)+\log _{2} e .
\end{aligned}
$$

Note that (45) states that the optimum compressed length is below the entropy. This is a consequence of lifting the prefix condition: without prefix constraints one can compress at an average rate slightly below the entropy [52], [54].

The operational rate-cost function for control under variablelength quantization, $R_{\mathrm{var}}(b)$, is defined as the limsup of the infimum of $r$ 's such that LQR cost $b$ and (44) are achievable in the limit of large $t$. It follows from (45) that

$$
R_{\text {var }}(b) \leq \mathbb{H}(b),
$$

which implies the existence of a variable-length quantizer whose average rate does not exceed the expressions in Theorems 2 and 6. Likewise, by (46) and Jensen's inequality,

$$
\mathbb{H}(b) \leq R_{\mathrm{var}}(b)+\log _{2}\left(R_{\mathrm{var}}(b)+1\right)+\log _{2} e,
$$

which leads via (18) to the lower bound $R_{\mathrm{var}}(b) \geq \psi^{-1}(\mathbb{R}(b)$ ), where $\psi^{-1}(\cdot)$ is the functional inverse of $\psi(x)=x+$ $\log _{2}(x+1)+\log _{2} e$. Thus, Theorems 1, 3, 4, 5, 7 and 8 provide lower bounds to $R_{\mathrm{var}}(b)$. Consequently, our converse and achievability results in Section II-A and Section II-B characterize the operational rate-cost tradeoff for control with variable-length quantizers.

Remark 4. The minimum average compressed length among all prefix-free lossless compressors, $L_{\mathrm{p}}^{\star}(X)$, satisfies $H(X) \leq$ $L_{\mathrm{p}}^{\star}(X) \leq H(X)+1$. Therefore, the minimum average rate of a prefix-free quantizer compatible with cost $b$ is bounded as $\mathbb{R}(b) \leq R_{\text {var }, \mathrm{p}}(b) \leq \mathbb{H}(b)+1$. Accordingly, the theorems in Section II-A and Section II-B also characterize the operational rate-cost tradeoff for control with variable-length prefix-free quantizers.

\section{CAusal Shannon lower Bound}

In this section, we consider causal compression of a discretetime random process, $S^{\infty}=\left(S_{1}, S_{2}, \ldots\right)$, under the weighted mean-square error (MSE):

$\operatorname{WMSE}\left(S^{t}, \hat{S}^{t}\right) \triangleq \sum_{i=1}^{t} \mathbb{E}\left[\left(S_{i}-\hat{S}_{i}\right)^{T} \mathrm{~W}_{i}\left(S_{i}-\hat{S}_{i}\right)\right]$,

where $\mathrm{W}_{i} \geq 0$. Understanding causal compression bears great independent interest and, due to separation between quantization and control, it is also vital to understanding quantized control, as explained in Section IV below.

Causally conditioned directed information and causally conditioned entropy are defined as:

$$
\begin{gathered}
I\left(S^{t} \rightarrow \hat{S}^{t} \| Z^{t}\right) \triangleq \sum_{i=1}^{t} I\left(S^{i} ; \hat{S}_{i} \mid \hat{S}^{i-1}, Z^{i}\right), \\
H\left(\hat{S}^{t} \| Z^{t}\right) \triangleq \sum_{i=1}^{t} H\left(\hat{S}_{i} \mid \hat{S}^{i-1}, Z^{i}\right) .
\end{gathered}
$$

Definition 4 (causal rate- and entropy-distortion functions). The causal rate- and entropy-distortion functions under the weighted MSE in (49) with side information $Z^{\infty}$ causally available at both encoder and decoder are defined as,

$$
\begin{aligned}
& \mathbb{R}_{S^{\infty} \| Z^{\infty}}(d) \triangleq \limsup _{t \rightarrow \infty} \inf _{P_{\hat{S}^{t} \| S^{t}, Z^{t}}:} \frac{1}{t} \operatorname{WMSE}\left(S^{t}, \hat{S}^{t}\right) \leq d \\
& \mathbb{H}_{S^{\infty} \| Z^{\infty}}(d) \triangleq \limsup _{t \rightarrow \infty} \inf _{\substack{P_{\hat{S}^{t} \| S^{t}, Z^{t}}: \\
\frac{1}{t} \operatorname{WMSE}\left(S^{t}, \hat{S}^{t}\right) \leq d}} \frac{1}{t} H\left(\hat{S}^{t} \| Z^{t}\right) .
\end{aligned}
$$

In the absence of side information, we write $\mathbb{R}_{S \infty}(d)$ / $\mathbb{H}_{S \infty}(d)$ for the causal rate- / entropy-distortion functions.

DPCM encoder, upon observing the current source sample $S_{i}$, computes the state innovation $\tilde{S}_{i}$ recursively using

$$
\tilde{S}_{i} \triangleq S_{i}-\hat{S}_{i \mid i-1}
$$

where $\hat{S}_{i \mid i-1} \triangleq \mathbb{E}\left[S_{i} \mid \hat{S}^{i-1}\right]$ is the a priori (predicted) state estimate at the decoder given previous decoder's outputs $\hat{S}^{i-1}$. The DPCM encoder sends quantized innovation $\hat{\tilde{S}}_{i}$.

DPCM decoder, having recovered $\hat{\tilde{S}}_{i}$, forms its estimate:

$$
\hat{S}_{i}=\hat{S}_{i \mid i-1}+\hat{\tilde{S}}_{i}
$$

Proposition 2 below implies that the causal rate- and entropy-distortion functions are attained in the class of DPCM (let $T_{i}=-\hat{S}_{i \mid i-1}$ in Proposition 2). No independence among samples of either the innovation process $\left\{\tilde{S}_{i}\right\}$ or its encoded version $\left\{\hat{\tilde{S}}_{i}\right\}$ is required for this to hold.

Proposition 2. Let the stochastic process $\left\{T_{i}\right\}$ be adapted to the filtration generated by $\left\{Z^{i}\right\}$. Then,

$$
\begin{aligned}
& \mathbb{R}_{S^{\infty} \| Z^{\infty}}(d)=\mathbb{R}_{S^{\infty}+T^{\infty} \| Z^{\infty}}(d), \\
& \mathbb{H}_{S^{\infty} \| Z^{\infty}}(d)=\mathbb{H}_{S^{\infty}+T^{\infty} \| Z^{\infty}}(d) .
\end{aligned}
$$

Proof. Since mutual information, entropy and distortion measure (49) are all invariant to shifts and $T_{i}$ is a common knowledge to both encoder and decoder at time $i, I\left(S^{t} \rightarrow \hat{S}^{t} \| Z^{t}\right)=$ $I\left(S^{t}+T^{t} \rightarrow \hat{S}^{t}+T^{t} \| Z^{t}\right), H\left(\hat{S}^{t} \| Z^{t}\right)=H\left(\hat{S}^{t}+T^{t} \| Z^{t}\right)$, $\operatorname{WMSE}\left(S^{t}, \hat{S}^{t}\right)=\mathrm{WMSE}\left(S^{t}+T^{t}, \hat{S}^{t}+T^{t}\right)$, and (56), (57) follow.

Proposition 2 implies further that the rate-distortion tradeoffs for controlled and uncontrolled processes are the same. Indeed, consider the Markov process

$$
S_{i+1}=\mathrm{A} S_{i}+V_{i}
$$

obtained by letting $U_{i} \equiv 0, i=1,2, \ldots$ in (1). The uncontrolled process (58) and the controlled one (1) are related through $X_{i}=S_{i}+\sum_{j=1}^{i-1} \mathrm{~A}^{i-1-j} \mathrm{~B} U_{j}$. Provided that the encoder and the decoder both have access to past controls, Proposition 2 with $T_{i}=\sum_{j=1}^{i-1} \mathrm{~A}^{i-1-j} \mathrm{~B} U_{j}$ yields

$$
\begin{aligned}
& \mathbb{R}_{S^{\infty}}(d)=\mathbb{R}_{X^{\infty} \| \mathcal{D} U^{\infty}}(d), \\
& \mathbb{H}_{S \infty}(d)=\mathbb{H}_{X^{\infty} \| \mathcal{D} U^{\infty}}(d),
\end{aligned}
$$

where $\mathcal{D} U^{\infty}$ signifies causal availability of past controls. The choice of these controls does not affect the rate-distortion tradeoffs in (59) and (60). Furthermore, using $\hat{S}_{i \mid i-1}=\mathrm{A} \hat{S}_{i-1}$, $\hat{X}_{i \mid i-1}=\mathrm{A} \hat{X}_{i-1}+\mathrm{B} U_{i-1}$, it is easy to show that both processes (58) and (1) have the same innovation process: 
$\tilde{X}_{i}=\tilde{S}_{i}$. Thus the same DPCM scheme can be used to encode both.

The following bound is a major result of this paper.

Theorem 9. For the Markov process (58) and a sequence of weight matrices such that

$$
\lim _{i \rightarrow \infty}\left(\operatorname{det} \mathrm{W}_{i}\right)^{\frac{1}{n}}=w>0,
$$

causal rate-distortion function is bounded below as

$$
\mathbb{R}_{S^{\infty}}(d) \geq \frac{n}{2} \log \left(a^{2}+\frac{w N(V)}{d / n}\right),
$$

where

$$
a \triangleq|\operatorname{det} \mathrm{A}|^{\frac{1}{n}} \text {. }
$$

The proof of Theorem 9 uses the classical Shannon lower bound, together with a dynamic programming argument. Theorem 9 can thereby be viewed as an extension of Shannon's lower bound to the causal compression setting.

Remark 5. For scalar Gauss-Markov sources, equality holds in (62) as long as the expression under the $\log$ is $\geq 1$, recovering the known result [2], [23], [32, Th. 3]

$$
\mathbb{R}_{S^{\infty}}(d)=\frac{1}{2}\left|\log \left(a^{2}+\frac{w \operatorname{Var}[V]}{d}\right)\right|_{+} .
$$

Remark 6. In lieu of the long-term average (Cesàro mean) constraint in (49), [2], [23] considered a more stringent constraint in which the average distortion is bounded at each time instant $i$. In the infinite time horizon, for Gauss-Markov sources, both formulations are equivalent, as optimal rates and distortions settle down to their steady states [32, Th. 3], [55]. For the scalar case this equivalence also follows by comparing (62) (obtained with a Cesàro mean constraint) and (64) (obtained in [2], [23] with a pointwise constaint).

Theorem 10, stated next, shows that the converse in Theorem 9 can be approached by a DPCM quantization scheme with uniform rate and distortion allocations (in the limit of infinite time horizon). This implies that nonuniform rate and distortion allocations permitted by (49) and (50)-(51) cannot yield significant performance gains.

Theorem 10. For the Markov process (58) such that $V$ has a regular density and a sequence of weight matrices such that (61) holds, the causal entropy function is bounded by

$\mathbb{H}_{S^{\infty}}(d) \leq \frac{n}{2} \log \left(a^{2}+\frac{w N(V)}{d / n}\right)+O_{1}(\log n)+O_{2}\left(d^{\frac{1}{2}}\right)$,

where $O_{1}(\log n) \leq C_{1} \log n$ and $O_{2}(\xi) \leq C_{2} \min \left\{\xi, c_{2}\right\}$ for some nonnegative constants $C_{1}, C_{2}$ and $c_{2}$. Furthermore, (65) is attained by a DPCM quantizer with output $\hat{S}_{i}$ such that $\lim _{i \rightarrow \infty} \mathbb{E}\left[\left(S_{i}-\hat{S}_{i}\right)^{T} \mathrm{~W}_{i}\left(S_{i}-\hat{S}_{i}\right)\right] \leq d$ and $\lim _{t \rightarrow \infty} H\left(\hat{S}_{i} \mid \hat{S}^{i-1}\right) \leq$ the right side of (65).

We conclude Section III with two extensions of Theorem 9. If the dynamic range of the eigenvalues of $A$ is large, the bound in Theorem 11 below, obtained by projecting out a subset of smaller eigenvalues of $A$, can provide an improvement over Theorem 9 at medium to large $d$. Recall the definition of matrices $\mathrm{J}, \Pi_{\ell}$ in (21), (22), respectively.
Theorem 11. Consider the uncontrolled process (58) and the distortion in (49). Consider matrices $\bigvee_{i}$ such that $0 \preceq \bigvee_{i}^{T} \bigvee_{i} \preceq$ $\mathrm{J}^{T} \mathrm{~W}_{i} \mathrm{~J}$, and $\mathrm{V}_{i}$ commutes with $\Pi_{\ell} \Pi_{\ell}^{T}$. Assume that

$$
w^{\prime} \triangleq \liminf _{i \rightarrow \infty}\left(\operatorname{det}\left(\Pi_{\ell}^{T} \mathrm{~V}_{i}^{T} \mathrm{~V}_{i} \Pi_{\ell}\right)\right)^{\frac{1}{m}}>0
$$

The causal rate-distortion function is bounded below as,

$$
\mathbb{R}_{S \infty}(d) \geq \frac{\ell}{2} \log \left(a^{\prime 2}+\frac{w^{\prime} N\left(\Pi_{\ell}^{T} \mathrm{~J}^{-1} V\right)}{d / \ell}\right)
$$

where $a^{\prime}$ is defined in (24).

Theorem 11 implies that $\mathbb{R}_{S^{\infty}}(d)$ is bounded as in (26). If $\mathrm{W}_{i}$ is singular, or if $V_{i}$ does not have a density with respect to the Lebesgue measure on $\mathbb{R}^{n}$, the bounds in Theorem 9 and 11 reduce to $n \log a$ and $n \log a^{\prime}$, respectively, losing the dependence on $d$. The bound in Theorem 12 below is a decreasing function of $d$, even if $\mathrm{W}_{i}$ is singular, or if $V_{i}$ is supported on a subspace of $\mathbb{R}^{n}$.

Theorem 12. Consider the uncontrolled process (58) and the distortion in (49). Assume that the weight matrices $\mathrm{W}_{i}$ satisfy $\lim _{i \rightarrow \infty} \mathrm{W}_{i}=\mathrm{L}^{T} \mathrm{~L}$, where $\mathrm{L}$ is an $m \times n$ matrix, $m \leq n$. Suppose further that $V_{i}=\mathrm{K}_{i} V_{i}^{\prime}$, where $V_{i}^{\prime}$ is a $k$-dimensional random vector with covariance matrix $\Sigma_{V^{\prime}}$, where $k \geq m$, and $\mathrm{K}_{i}$ are $n \times k$ matrices such that $\lim _{i \rightarrow \infty} \mathrm{K}_{i}=\mathrm{K}$. The causal rate-distortion function is bounded below as

$$
\mathbb{R}_{S \infty}(d) \geq \frac{m}{2} \log \left(a^{2}+\frac{w N\left(V^{\prime}\right)^{\frac{k}{m}}}{d / m}\right)
$$

$$
\text { where } \quad \begin{aligned}
a & =\inf _{i \geq 1}\left(\frac{\operatorname{det}\left(\mathrm{LA}^{i} \mathrm{~K} \Sigma_{V^{\prime}} \mathrm{K}^{T} \mathrm{~A}^{i T} \mathrm{~L}^{T}\right)}{\operatorname{det} \Sigma_{V^{\prime}} \operatorname{det}\left(\mathrm{LL}^{T}\right) \operatorname{det}\left(\mathrm{K}^{T} \mathrm{~K}\right)}\right)^{\frac{1}{2 i m}} \\
w & =\left(\operatorname{det}\left(\mathrm{LL}^{T}\right) \operatorname{det}\left(\mathrm{K}^{T} \mathrm{~K}\right)\right)^{\frac{1}{m}}
\end{aligned}
$$

If $k=m=n$, Theorem 12 reduces to Theorem 9 .

\section{CONTROL, ESTIMATION AND COMMUNICATION SEPARATED}

An early quantization-control separation result for Gaussian systems was proposed by Fischer [56]. Tatikonda et al. [2] considered control of fully observed system over a noisy channel and showed that certainty equivalence control is optimal if and only if control has no dual effect, that is to say the present control cannot affect the future state uncertainty. Here, we observe that as long as both the encoder and the controller have access to past controls, separated design for control over noisy channels is optimal, both for fully observed systems and for Gaussian partially observed systems.

Recall the last-step weight matrix $S_{t}$ in (3), and let $S_{i}, 1 \leq$ $i \leq t-1$ be the solution to the Riccati recursion,

$$
\mathrm{S}_{i}=\mathrm{Q}+\mathrm{A}^{T}\left(\mathrm{~S}_{i+1}-\mathrm{M}_{i}\right) \mathrm{A} \text {. }
$$

where

$$
\begin{aligned}
\mathrm{M}_{i} & \triangleq \mathrm{S}_{i+1} \mathrm{~B}\left(\mathrm{R}+\mathrm{B}^{T} \mathrm{~S}_{i+1} \mathrm{~B}\right)^{-1} \mathrm{~B}^{T} \mathrm{~S}_{i+1} \\
& =\mathrm{L}_{i}^{T}\left(\mathrm{R}+\mathrm{B}^{T} \mathrm{~S}_{i+1} \mathrm{~B}\right) \mathrm{L}_{i} \\
\mathrm{~L}_{i} & \triangleq\left(\mathrm{R}+\mathrm{B}^{T} \mathrm{~S}_{i+1} \mathrm{~B}\right)^{-1} \mathrm{~B}^{T} \mathrm{~S}_{i+1}
\end{aligned}
$$

The optimal control of partially observed system in (1), (2) in the scenario of Fig. 1, for a fixed dynamical channel $\left\{P_{G_{i} \mid G^{i-1}, F^{i}}\right\}_{i=1}^{\infty}$ can be obtained as follows: 
(i) The encoder computes the optimal state estimate

$$
\hat{X}_{i} \triangleq \mathbb{E}\left[X_{i} \mid Y^{i}, U^{i-1}\right] .
$$

(ii) The encoder maps the optimal control signal $U_{i}^{\star}=$ $-\mathrm{L}_{i} \mathrm{~A} \hat{X}_{i}$ to channel codeword $F_{i}$;

(iii) Having received the channel output $G_{i}$, the decoder computes $U_{i}$ and applies it to the system.

The encoder $\left\{P_{F_{i} \mid Y^{i}, U^{i-1}}\right\}$ and the controller $\left\{P_{U_{i} \mid G^{i}, U^{i-1}}\right\}$ are designed to minimize the LQR cost, written as follows.

Theorem 13. The LQR cost in the scenario of Fig. 1 for the partially observed system (1), (2) separates as,

$$
\operatorname{LQR}\left(X^{t}, U^{t-1}\right)=\sum_{i=0}^{t-1} c_{i}+\sum_{i=1}^{t-1} e_{i}+\sum_{i=1}^{t-1} d_{i},
$$

where the control, estimation and communication costs are respectively given by

$$
\begin{aligned}
c_{i} & \triangleq \operatorname{tr}\left(\Sigma_{V} \mathrm{~S}_{i+1}\right), \quad c_{0} \triangleq \operatorname{tr}\left(\Sigma_{X_{1}} \mathrm{~S}_{1}\right) \\
e_{i} & \triangleq \mathbb{E}\left[\left(X_{i}-\hat{X}_{i}\right)^{T} \mathrm{~A}^{T} \mathrm{M}_{i} \mathrm{~A}\left(X_{i}-\hat{X}_{i}\right)\right], \\
d_{i} & \triangleq \mathbb{E}\left[\left(U_{i}-U_{i}^{\star}\right)^{T}\left(\mathrm{R}+\mathrm{B}^{T} \mathrm{~S}_{i+1} \mathrm{~B}\right)\left(U_{i}-U_{i}^{\star}\right)\right] .
\end{aligned}
$$

Proof. Denote for $1 \leq i \leq t$

$$
b_{i} \triangleq \mathbb{E}\left[\sum_{j=1}^{i-1}\left(X_{j}^{T} \mathrm{Q} X_{j}+U_{j}^{T} \mathrm{R} U_{j}\right)\right]+\mathbb{E}\left[X_{i}^{T} \mathrm{~S}_{i} X_{i}\right] .
$$

Eliminating $X_{i}$ from (80) by substituting $X_{i}=\mathrm{A} X_{i-1}+$ $\mathrm{B} U_{i-1}+V_{i-1}$ into (80), completing the squares and using that $V_{i-1}$ is zero-mean and independent of $X_{i-1}, U_{i-1}$, we re-write (80) equivalently as

$$
b_{i}=b_{i-1}+\mathbb{E}\left[V_{i-1}^{T} \mathrm{~S}_{i} V_{i-1}\right]+q_{i-1},
$$

where

$$
q_{i} \triangleq \mathbb{E}\left[\left(\mathrm{L}_{i} \mathrm{~A} X_{i}+U_{i}\right)^{T}\left(\mathrm{R}+\mathrm{B}^{T} \mathrm{~S}_{i+1} \mathrm{~B}\right)\left(\mathrm{L}_{i} \mathrm{~A} X_{i}+U_{i}\right)\right]
$$

Applying (81) repeatedly, we obtain

$$
b_{t}=b_{1}+\sum_{i=1}^{t-1} \mathbb{E}\left[V_{i}^{T} S_{i+1} V_{i}\right]+\sum_{i=1}^{t-1} q_{i},
$$

which is equivalent to (76) in the fully observed scenario, i.e. when $\hat{X}_{i}=X_{i}$.

To show the more general case, observe first that for random vectors $X, Y$ and $\hat{X}$ forming a Markov chain $X-Y-\hat{X}$, it holds with $\hat{X}^{\star} \triangleq \mathbb{E}[X \mid Y]$ that,

$$
\mathbb{E}\left[\|X-\hat{X}\|^{2}\right]=\mathbb{E}\left[\left\|X-\hat{X}^{\star}\right\|^{2}\right]+\mathbb{E}\left[\left\|\hat{X}^{\star}-\hat{X}\right\|^{2}\right] .
$$

Applying (84) to $\mathrm{L}_{i} \mathrm{~A} X_{i}-\left(Y^{i}, U^{i-1}\right)-U_{i}$, we separate $q_{i}$ into $q_{i}=e_{i}+d_{i}$, and thereby rewrite (83) as (76).

Using Theorem 13, we write the minimum achievable LQR cost as

$$
\begin{aligned}
& \sum_{i=0}^{t-1} c_{i}+\inf \left\{\sum_{i=1}^{t-1} e_{i}+\sum_{i=1}^{t-1} d_{i}\right\} \\
\geq & \sum_{i=0}^{t-1} c_{i}+\inf \left\{\sum_{i=1}^{t-1} e_{i}\right\}+\inf \left\{\sum_{i=1}^{t-1} d_{i}\right\},
\end{aligned}
$$

where the infimum is over all admissible control sequences. Equality in (85) is not attained in general; two important scenarios when is it achieved are fully observed systems and for Gaussian partially observed systems. In the fully observed case, the estimation terms $e_{i}$ disappear, and in the Gaussian partially observed case, it is well known that those terms do not depend on the choice of controls. Indeed, if $V_{i}$ and $W_{i}$ are both Gaussian, then the optimal estimator (75) can be implemented via a linear recursion given by the Kalman filter. At time $i$, having observed $Y_{i}$, the Kalman filter forms an estimate of the system state using $Y_{i}$ and the prior estimate $\hat{X}_{i-1}$ as follows (e.g. [57]):

$$
\hat{X}_{i}=\mathrm{A} \hat{X}_{i-1}+\mathrm{B} U_{i-1}+\mathrm{K}_{i} \tilde{Y}_{i}
$$

where

$$
\tilde{Y}_{i} \triangleq Y_{i}-\mathrm{CA} \hat{X}_{i-1}-\mathrm{CB} U_{i-1}
$$

$\mathrm{y}$ is the innovation, Gaussian and independent of $\hat{X}_{i-1}, U_{i-1}$ and $\tilde{Y}^{i-1}$, and the Kalman filter gain is found from the Riccati recursion:

$$
\begin{aligned}
\mathrm{K}_{i} & \triangleq \mathrm{P}_{i \mid i-1} \mathrm{C}^{T}\left(\mathrm{CP}_{i \mid i-1} \mathrm{C}^{T}+\Sigma_{W}\right)^{-1}, \\
\mathrm{P}_{i+1 \mid i} & =\mathrm{A}\left(\mathrm{I}-\mathrm{K}_{i} \mathrm{C}\right) \mathrm{P}_{i \mid i-1} \mathrm{~A}^{T}+\Sigma_{V}, \quad \mathrm{P}_{1 \mid 0} \triangleq \Sigma_{X_{1}} .
\end{aligned}
$$

The covariances of the innovation and the estimation error are given by, respectively,

$$
\begin{aligned}
\operatorname{Cov}\left(\tilde{Y}_{i}\right) & =\mathrm{CP}_{i \mid i-1} \mathrm{C}^{T}+\Sigma_{W}, \\
\operatorname{Cov}\left(X_{i}-\hat{X}_{i}\right) & =\left(\mathrm{I}-\mathrm{K}_{i} \mathrm{C}\right) \mathrm{P}_{i \mid i-1},
\end{aligned}
$$

and the estimation error is thus given by

$$
\left.e_{i}=\operatorname{tr}\left(\left(\mathrm{I}-\mathrm{K}_{i} \mathrm{C}\right) \mathrm{P}_{i \mid i-1}\right) \mathrm{A}^{T} \mathrm{M}_{i} \mathrm{~A}\right) .
$$

An immediate corollary to Theorem 13 is the following.

Corollary 1. In both fully observed systems and Gaussian partially observed systems, the rate-cost and the entropy-cost functions and independent of the control sequence $U^{\infty}$ and are given by, respectively,

$$
\begin{aligned}
& \mathbb{R}(b)=\mathbb{R}_{\hat{X}^{\infty} \| \mathcal{D} U^{\infty}}\left(b-b_{\text {min }}\right), \\
& \mathbb{H}(b)=\mathbb{H}_{\hat{X}^{\infty} \| \mathcal{D} U^{\infty}}\left(b-b_{\text {min }}\right),
\end{aligned}
$$

where $b_{\min }$ is the minimum cost attainable without communication constraints in (30), and causal rate- and entropydistortion functions are evaluated with weight matrices

$$
\mathrm{W}_{i}=\mathrm{A}^{T} \mathrm{M}_{i} \mathrm{~A} .
$$

Proof. Since equality in (85) holds, we need to minimize $\sum_{i=1}^{t-1} d_{i}$ subject to either directed information or entropy constraint. Once we argue that the minimal achievable distortions can be equivalently written as

$$
d_{i}=\mathbb{E}\left[\left(\hat{X}_{i}-\hat{X}_{i}\right)^{T} \mathrm{~A}^{T} \mathrm{M}_{i} \mathrm{~A}\left(\hat{X}_{i}-\hat{X}_{i}\right)\right],
$$

where $\hat{X}_{i}$ is the controller's estimate of $\hat{X}_{i}$, and $U_{i}=-\mathrm{L}_{i} \mathrm{~A} \hat{X}_{i}$, we will immediately obtain (93), (94). But this follows via the same arguments as in the proof of Proposition 6 below, using data processing for directed information [58, Lemma 4.8.1] in lieu of that for mutual information.

Via Corollary 1, we can show the converse for control over noisy channels in Proposition 1 using a converse for tracking over noisy channels. Tracking $S_{1}, S_{2}, \ldots$ over a causal feedback channel $P_{G^{t} \| F^{t}}$ gives rise to a joint distribution of the form $P_{S^{t}} P_{F^{t} \| S^{t}, \mathcal{D} G^{t}} P_{G^{t} \| F^{t}} P_{\hat{S}^{t} \| G^{t}}$, where $P_{F^{t} \| S^{t}, \mathcal{D} G^{t}}$ and $P_{\hat{S}^{t} \| G^{t}}$ represent encoder and decoder mappings, and the goal is to minimize the distortion between $S^{t}$ and $\hat{S}^{t}$. A necessary condition for the existence of an encoder/decoder pair achieving distortion $d$ in the limit of infinite time horizon is [58, Th. 5.3.2],

$$
\mathbb{R}_{S \infty}(d) \leq C .
$$


Proposition 1 follows by plugging (59) and (93) in (97).

\section{CONVERSE THEOREMS: TOOLS AND PROOFS}

We start by introducing a few definitions and tools, some classical, some novel, that form the basis of our technique.

Conditional entropy power is defined as

$$
N(X \mid U) \triangleq \frac{1}{2 \pi e} \exp \left(\frac{2}{n} h(X \mid U)\right),
$$

where $h(X \mid U)=-\mathbb{E}\left[\int_{\mathbb{R}^{n}} f_{X \mid U}(x \mid U) \log f_{X \mid U}(x \mid U) d x\right]$ is the conditional differential entropy of $X$.

Proposition 3. For $X \in \mathbb{R}^{n}$,

$$
n N(X \mid U) \leq \operatorname{Var}[X \mid U]
$$

with equality if and only if $X=U+S$, where $S$ is Gaussian.

Proof. The unconditional case is a well-known maximum entropy result (e.g. [59, Example 12.2.8]). This implies that for each realization of $u, n N(X \mid U=u) \leq \operatorname{Var}[X \mid U=u]$. Taking expectation with respect to $U$ of both sides and using strict convexity of $x \mapsto \exp (x)$, we obtain (99) together with condition for equality.

An essential component of our analysis, the conditional entropy power inequality (EPI), follows from the unconditional EPI [36], [37] using convexity of the function $(x, y) \mapsto$ $\log (\exp (x)+\exp (y))$.

Theorem 14 (Conditional EPI). If $X \Perp Y$ given $U$, then

$$
N(X+Y \mid U) \geq N(X \mid U)+N(Y \mid U) \text {. }
$$

In causal data compression, the quantized data at current step creates the side information for the data to be compressed at the next step. The following bound to the conditional entropy power minimized over side information will be vital in proving our converse theorems.

Proposition 4. For $X \in \mathbb{R}^{n}$,

$$
\inf _{P_{U \mid X}: I(X ; U) \leq r} N(X \mid U) \geq N(X) \exp (-2 r / n) .
$$

Proof. Observe that

$$
\begin{aligned}
\inf _{P_{U \mid X}: I(X ; U) \leq r} h(X \mid U) & =\inf _{P_{U \mid X}: h(X)-h(X \mid U) \leq r} h(X \mid U) \\
& \geq h(X)-r,
\end{aligned}
$$

which is equivalent to (101).

If $L$ is square, the entropy power scales as

$$
N(\mathrm{~L} X \mid U)=|\operatorname{det} \mathrm{L}|^{\frac{2}{n}} N(X \mid U) .
$$

The next proposition generalizes the scaling property (103) to the case where the multiplying matrix is not square. ${ }^{6}$

Proposition 5. Let $X \in \mathbb{R}^{n}$ be a random vector with covariance $\Sigma_{X} \succ 0$, let $m \leq n$, and let $\mathrm{L}$ be an $m \times n$ matrix with rank $m$. Then,

$$
N(\mathrm{~L} X) \geq\left(\frac{\operatorname{det}\left(\mathrm{L} \Sigma_{X} \mathrm{~L}^{T}\right)}{\operatorname{det} \Sigma_{X}}\right)^{\frac{1}{m}}(N(X))^{\frac{n}{m}}
$$

\footnotetext{
${ }^{6}$ Proposition 5 is stated for the unconditional case for simplicity only; naturally, its conditional version also holds.
}

Equality holds in (104) if $m=n$ or if $X$ is Gaussian.

Proof. Without loss of generality, assume $\mathbb{E}[X]=0$. Express $h(X)$ through the relative entropy $D(\cdot \| \cdot)$ as

$h(X)=\frac{1}{2} \log \left((2 \pi e)^{n} \operatorname{det} \Sigma_{X}\right)-D\left(P_{X} \| \mathcal{N}\left(\mathbf{0}, \Sigma_{X}\right)\right)$,

By the data processing inequality of relative entropy,

$$
\begin{aligned}
& D\left(P_{X} \| \mathcal{N}\left(\mathbf{0}, \Sigma_{X}\right)\right) \geq D\left(P_{\mathrm{L} X} \| \mathcal{N}\left(\mathbf{0}, \mathrm{L} \Sigma_{X} \mathrm{~L}^{T}\right)\right) \\
= & \frac{1}{2} \log \left((2 \pi e)^{m} \operatorname{det}\left(\mathrm{L} \Sigma_{X} \mathrm{~L}^{T}\right)\right)-h(\mathrm{~L} X),
\end{aligned}
$$

and (104) follows by substituting (107) into (105) and applying (12).

The (single-shot) distortion-rate function with respect to the weighted mean-square distortion is defined as follows.

Definition 5 (conditional distortion-rate function). Let $X \in$ $\mathbb{R}^{n}$ be a random vector, and $\mathrm{M} \succeq 0$ be an $n \times n$ matrix. The distortion-rate function under the weighted MSE with side information $U$ at both the encoder and the decoder is

$$
\mathbb{D}_{r, \mathrm{M}}(X \mid U) \triangleq \inf _{\substack{P_{\hat{X} \mid X U}: \\ I(X ; \hat{X} \mid U) \leq r}} \mathbb{E}\left[(X-\hat{X})^{T} \mathrm{M}(X-\hat{X})\right] .
$$

If no side information is available, i.e. $U \equiv 0$, we denote the corresponding unconditional distortion-rate function by $\mathbb{D}_{r, \mathrm{M}}(X)$. The distortion-rate function under MSE distortion corresponds to $\mathrm{M}=\mathrm{I}$, and we simply denote

$$
\mathbb{D}_{r}(X \mid U) \triangleq \mathbb{D}_{r, \mathrm{I}}(X \mid U) .
$$

The next proposition equates the distortion-rate functions under weighted and non-weighed MSE.

Proposition 6. Let $X \in \mathbb{R}^{n}$ be a random vector, and let $\mathrm{L}$ be an $m \times n$ matrix. The following equality holds.

$$
\mathbb{D}_{r}(\mathrm{~L} X \mid U)=\mathbb{D}_{r, \mathrm{~L} T} \mathrm{~L}(X \mid U) .
$$

Proof. We show the unconditional version of (110); the conditional one is analogous. We will prove

$$
\begin{aligned}
\mathbb{D}_{r}(\mathrm{~L} X) & \triangleq \inf _{\hat{X}: I(\mathrm{~L} X ; \hat{X}) \leq r} \mathbb{E}\left[(\mathrm{L} X-\hat{X})^{T}(\mathrm{~L} X-\hat{X})\right] \\
& =\inf _{\hat{X}: I(\mathrm{~L} X ; \mathrm{L} \hat{X}) \leq r} \mathbb{E}\left[(X-\hat{X})^{T} \mathrm{~L}^{T} \mathrm{~L}(X-\hat{X})\right] \\
& =\inf _{\hat{X}: I(X ; \hat{X}) \leq r} \mathbb{E}\left[(X-\hat{X})^{T} \mathrm{~L}^{T} \mathrm{~L}(X-\hat{X})\right] \\
& \triangleq \mathbb{D}_{r, \mathrm{~L} T \mathrm{~L}(X) .} .
\end{aligned}
$$

To show $\geq$ in (112), let $\Pi$ be the orthogonal projection matrix onto the column space of $\mathrm{L}$. We use $\|\Pi x\| \leq\|x\|$ and $\Pi \mathrm{L} x=\mathrm{L} x$ to claim $\mathbb{E}\left[(\mathrm{L} X-\hat{X})^{T}(\mathrm{~L} X-\hat{X})\right] \geq$ $\mathbb{E}\left[(\mathrm{L} X-\Pi \hat{X})^{T}(\mathrm{~L} X-\Pi \hat{X})\right]$, and data processing for mutual information to claim $I(\mathrm{~L} X ; \hat{X}) \geq I(\mathrm{~L} X ; \Pi \hat{X})$. Likewise, $\leq$ holds in (113) by data processing. To show that $\leq$ holds in (112), we note that the optimization problem in (112) is obtained by restricting the domain of minimization in (111) to $\hat{X} \in \operatorname{Im}(\mathrm{L})^{7}$. To show that $\geq$ holds in (113), we note that the optimization problem in (112) is obtained by restricting the domain of minimization in (113) to $\hat{X} \in \operatorname{Im}(\mathrm{L})$ satisfying

\footnotetext{
${ }^{7}$ The image of a linear transformation described by matrix $L$ is the span of its column vectors.
} 
the Markov chain condition $X-\mathrm{L} X-\hat{X}$, since for such $\hat{X}$, $I(X ; \hat{X})=I(\mathrm{~L} X ; \hat{X})=I(\mathrm{~L} X ; \mathrm{L} \hat{X})$.

Remark 7. We may always assume that $X$ has uncorrelated components when computing distortion-rate functions. Indeed, let $\mathrm{L}$ be the orthogonal transformation that diagonalizes the covariance matrix of $X$. Since $\mathrm{L}^{T} \mathrm{~L}=\mathrm{I}$, by Proposition 6 the MSE distortion-rate functions of $X$ and $\mathrm{L} X$ coincide.

The following tool will be instrumental in our analysis.

Theorem 15 (Conditional Shannon lower bound). The conditional distortion-rate function is bounded below as

$$
\mathbb{D}_{r}(X \mid U) \geq \underline{\mathbb{D}}_{r}(X \mid U) \triangleq n N(X \mid U) \exp (-2 r / n),
$$

with equality if $X=U+S$, where $S \sim \mathcal{N}\left(0, \sigma^{2} \mathrm{I}\right)$.

Proof. Theorem 15 is a conditional version of Shannon's lower bound [35]. Using Propositions 3 and 4, we can write, for any $Y$ such that $I(X ; Y \mid U) \leq r$,

$$
\begin{aligned}
\mathbb{E}\left[\|X-Y\|^{2}\right] & \geq \mathbb{E}\left[\|X-\mathbb{E}[X \mid Y, U]\|^{2}\right] \\
& \geq N(X \mid Y, U) \\
& \geq N(X \mid U) \exp (-2 r / n) .
\end{aligned}
$$

The equality condition is verified by checking that $Y$ such that $X=Y+Z$, where $Z \sim \mathcal{N}\left(0, \sigma^{2} \exp (-2 r / n) \mathrm{I}\right)$, attains equalities in (116)-(118).

Shannon's lower bound is equal to the distortion-rate function of a white Gaussian vector with the same differential entropy as the original vector. Although beyond Gaussian $X$, Shannon's lower bound is rarely attained with equality [60], it is approached at high rates [61]. The tightness of Shannon's lower bound at high rates is key to arguing that the bound in Theorem 1 can in fact be approached.

For convenience, we record the following result, which is an immediate corollary to Proposition 4.

Proposition 7. Let $X \in \mathbb{R}^{n}$ be a random vector. The following inequality holds:

$$
\min _{U \in \mathbb{R}^{n}: I(X ; U) \leq s} \mathbb{D}_{r}(X \mid U) \geq \underline{\mathbb{D}}_{r+s}(X) .
$$

Remark 8. If $X$ is Gaussian, then

$$
\min _{U \in \mathbb{R}^{n}: I(X ; U) \leq s} \mathbb{D}_{r}(X \mid U)=\mathbb{D}_{r+s}(X),
$$

and the minimum is attained by a Gaussian $U$. For nonGaussian $X, \geq$ holds in (120).

We are now equipped to prove our converse theorems.

Proof of Theorem 9. For any causal kernel $P_{\hat{S}^{t} \| S^{t}}$ induced by a code, denote the per-stage information rates

$$
r_{i} \triangleq I\left(S^{i} ; \hat{S}_{i} \mid \hat{S}^{i-1}\right)
$$

Using $r_{i} \geq I\left(S_{i} ; \hat{S}_{i} \mid \hat{S}^{i-1}\right)$, Shannon's lower bound (Theorem 15), Proposition 6 and (103), we lower-bound the distortion at step $i$ as

$$
\begin{aligned}
\mathbb{E}\left[\left(S_{i}-\hat{S}_{i}\right)^{T} \mathrm{~W}_{i}\left(S_{i}-\hat{S}_{i}\right)\right] & \geq \mathbb{D}_{r_{i}, \mathrm{~W}_{i}}\left(S_{i} \mid \hat{S}^{i-1}\right) \\
& \geq w_{i} \underline{d}_{i},
\end{aligned}
$$

where we denoted for brevity

$$
\underline{d}_{i} \triangleq \underline{\mathbb{D}}_{r_{i}}\left(S_{i} \mid \hat{S}^{i-1}\right), i=1,2, \ldots, t
$$

$$
w_{i} \triangleq\left(\operatorname{det} \mathrm{W}_{i}\right)^{\frac{1}{n}}
$$

Next, define $\underline{d}_{0}$ satisfy $a^{2} \underline{d}_{0}+n N(V)=n N\left(S_{1}\right)$. We establish the following recursion $(1 \leq i \leq t)$ :

$$
\begin{aligned}
\underline{d}_{i} & =\underline{\mathbb{D}}_{r_{i}}\left(\mathrm{~A} S_{i-1}+V_{i-1} \mid \hat{S}^{i-1}\right) \\
& \geq \underline{\mathbb{D}}_{r_{i}}\left(\mathrm{~A} S_{i-1} \mid \hat{S}^{i-1}\right)+\underline{\mathbb{D}}_{r_{i}}(V) \\
& \geq \underline{\mathbb{D}}_{r_{i-1}+r_{i}}\left(\mathrm{~A} S_{i-1} \mid \hat{S}^{i-2}\right)+\underline{\mathbb{D}}_{r_{i}}(V) \\
& =\left(a^{2} \underline{d}_{i-1}+n N(V)\right) \exp \left(-2 r_{i} / n\right)
\end{aligned}
$$

where (127) is by the conditional EPI (Theorem 14), (128) is due to (119). Note that (126)-(129) holds for an arbitrary encoded sequence $\hat{S}_{1}, \ldots, \hat{S}_{i-1}$, including the optimal one.

Rewriting (129) as

$$
2 r_{i} / n \geq \log \left(a^{2} \underline{d}_{i-1}+n N(V)\right)-\log \underline{d}_{i},
$$

we deduce

$$
\begin{aligned}
& \frac{1}{n} \sum_{i=1}^{t} r_{i} \geq \frac{1}{n} \sum_{i=t_{\epsilon}}^{t} r_{i} \geq \\
& \frac{1}{2} \log \frac{a^{2} \underline{d}_{t_{\epsilon}-1}+n N(V)}{a^{2} \underline{d}_{t}+n N(V)}+\frac{1}{2} \sum_{i=t_{\epsilon}}^{t} \log \left(a^{2}+\frac{N(V)}{\underline{d}_{i} / n}\right)
\end{aligned}
$$

where $t_{\epsilon}$ is defined for any $\epsilon>0$ as the smallest number such that for all $i \geq t_{\epsilon}, w_{i} \geq w-\epsilon$. Assumption (61) ensures that $t_{\epsilon}<\infty$. The distortion constraint in (52) and the bound (123) imply

$$
\frac{w-\epsilon}{t} \sum_{i=t_{\epsilon}}^{t} \underline{d}_{i} \leq \frac{1}{t} \sum_{i=1}^{t} w_{i} \underline{d}_{i} \leq d .
$$

In particular, (133) implies that $\underline{d}_{t} \leq d \frac{t}{w-\epsilon}$, which together with $a^{2} \underline{d}_{t_{\epsilon}-1}+n N(V) \geq n \min \left\{N\left(S_{1}\right), N(V)\right\}$ means that the first term in (132) normalized by $t$ is bounded below by a quantity that vanishes as $t \rightarrow \infty$.

Since the function $x \mapsto \log \left(a^{2}+\frac{n N(V)}{x}\right)$ is convex and decreasing, by Jensen's inequality and (133) the sum in (132) is bounded below as

$$
\begin{aligned}
& \sum_{i=t_{\epsilon}}^{t} \log \left(a^{2}+\frac{N(V)}{\underline{d}_{i} / n}\right) \\
\geq & \left(t-t_{\epsilon}\right) \log \left(a^{2}+\frac{(w-\epsilon) N(V)}{d / n} \frac{t-t_{\epsilon}}{t}\right),
\end{aligned}
$$

Diving both sides of (134) by $t$ and taking taking a $\lim _{t \rightarrow \infty}$ followed by a $\lim _{\epsilon \rightarrow 0}$, we obtain (62).

Proof of Theorem 11. We start by making two observations. First, putting $S_{i}^{\prime} \triangleq \mathrm{J}^{-1} S_{i}$ and $V_{i}^{\prime} \triangleq \mathrm{J}^{-1} V_{i}$, we may write

$$
S_{i+1}^{\prime}=\mathrm{A}^{\prime} S_{i}^{\prime}+V_{i}^{\prime},
$$

Causal rate-distortion functions of $S^{\infty}$ and $S^{\prime \infty}$ satisfy

$$
\mathbb{R}_{S^{\infty},\left\{\mathrm{W}_{i}\right\}}(d)=\mathbb{R}_{S^{\prime} \infty,\left\{\mathrm{J}^{T} \mathrm{~W}_{i} \mathrm{~J}\right\}}(d),
$$

where we indicated the weight matrices in the subscript.

Second, if $0 \preceq \Pi^{T} \Pi \preceq \mathrm{I}$, and $\Pi$ commutes with $\mathrm{L}$, then

$$
\Pi^{T} \mathrm{~L}^{T} \mathrm{~L} \sqcap \preceq \mathrm{L}^{T} \mathrm{~L} .
$$

Due to (136) we may focus on evaluating the rate-distortion function for $S^{\prime \infty}$. Since $\left(\Pi_{\ell} \Pi_{\ell}^{T}\right)^{2} \preceq \Pi_{\ell} \Pi_{\ell}^{T} \preceq \mathrm{I}$ and $\Pi_{\ell} \Pi_{\ell}^{T}$ commutes with $L_{i}$, we may apply (137) and Theorem 15 to obtain

$$
\mathbb{E}\left[\left(S_{i}^{\prime}-\hat{S}_{i}^{\prime}\right)^{T} \mathrm{~J}^{T} \mathrm{~W}_{i} \mathrm{~J}\left(S_{i}^{\prime}-\hat{S}_{i}^{\prime}\right)\right]
$$




$$
\begin{aligned}
& \geq \mathbb{E}\left[\left(S_{i}^{\prime}-\hat{S}_{i}^{\prime}\right)^{T} \Pi_{\ell} \Pi_{\ell}^{T} \mathrm{~J}^{T} \mathrm{~W}_{i} \mathrm{~J} \Pi_{\ell} \Pi_{\ell}^{T}\left(S_{i}^{\prime}-\hat{S}_{i}^{\prime}\right)\right] \\
& \geq w_{i}^{\prime} \underline{d}_{i}^{\prime},
\end{aligned}
$$

where

$$
\underline{d}_{i}^{\prime} \triangleq \underline{\mathbb{D}}_{r_{i}}\left(\Pi_{\ell}^{T} S_{i}^{\prime} \mid \hat{S}^{i-1}\right), i=1,2, \ldots, t .
$$

Since $\Pi_{\ell} \Pi_{\ell}^{T}$ commutes with $A^{\prime}$ and $\Pi_{\ell}^{T} \Pi_{\ell} \Pi_{\ell}^{T}=\Pi_{\ell}^{T}$,

$$
\Pi_{\ell}^{T} A^{\prime}=\Pi_{\ell}^{T} A^{\prime} \Pi_{\ell} \Pi_{\ell}^{T} .
$$

Using (142), Theorem 14 and Proposition 7, we establish

$$
\begin{aligned}
\underline{d}_{i}^{\prime} & =\underline{\mathbb{D}}_{r_{i}}\left(\Pi_{\ell}^{T} \mathrm{~A}^{\prime} S_{i-1}^{\prime}+\Pi_{\ell}^{T} V_{i-1}^{\prime} \mid \hat{S}^{i-1}\right) \\
& \geq \mathbb{D}_{r_{i}}\left(\Pi_{\ell}^{T} \mathrm{~A}^{\prime} S_{i-1}^{\prime} \mid \hat{S}^{i-1}\right)+\mathbb{D}_{r_{i}}\left(\Pi_{\ell}^{T} V_{i-1}^{\prime}\right) \\
& =\underline{\mathbb{D}}_{r_{i}}\left(\Pi_{\ell}^{T} \mathrm{~A}^{\prime} \Pi_{\ell} \Pi_{\ell}^{T} S_{i-1}^{\prime} \mid \hat{S}^{i-1}\right)+\underline{\mathbb{D}}_{r_{i}}\left(\Pi_{\ell}^{T} V_{i-1}^{\prime}\right) \\
& =a^{\prime 2} \underline{\mathbb{D}}_{r_{i}}\left(\Pi_{\ell}^{T} S_{i-1}^{\prime} \mid \hat{S}^{i-1}\right)+\mathbb{D}_{r_{i}}\left(\Pi_{\ell}^{T} V_{i-1}^{\prime}\right) \\
& \geq\left(a^{\prime 2} \underline{d}_{i-1}^{\prime}+m N\left(\Pi_{\ell}^{T} V^{\prime}\right)\right) \exp \left(-2 r_{i} / n\right)
\end{aligned}
$$

The rest of the proof follows that of Theorem 9 .

Proof of Theorem 12. It is easy to see (along the lines of (131)) that if $\mathrm{W}_{i} \rightarrow \mathrm{L}^{T} \mathrm{~L}$, we may put $\mathrm{W}_{i} \equiv \mathrm{L}^{T} \mathrm{~L}$ without affecting the (causal) rate-distortion function. Similarly, if $V_{i}=\mathrm{K}_{i} V_{i}^{\prime}$ and $\mathrm{K}_{i} \rightarrow \mathrm{K}$, we may put $V_{i}=\mathrm{K} V_{i}^{\prime}$. We will therefore focus on bounding the distortion with weight matrix $\mathrm{L}^{T} \mathrm{~L}$ and with $V_{i}=\mathrm{K} V_{i}^{\prime}$.

Adopting the convention $S_{1} \equiv V_{0}$, we rewrite (58) as,

$$
S_{i}=\sum_{j=0}^{i-1} \mathrm{~A}^{i-j-1} V_{j}
$$

Fixing causal reproduction vector $\hat{S}^{i}$, for $0 \leq j \leq i-1$, consider the random variable

$$
\tilde{V}_{j} \triangleq V_{j}-\mathbb{E}\left[V_{j} \mid \hat{S}^{i}\right]=V_{j}-\mathbb{E}\left[V_{j} \mid \hat{S}_{j+1}^{i}\right],
$$

where (149) holds because $V_{j}$ is independent of $\hat{S}^{j}$. Note that different $\tilde{V}_{j}$ 's are uncorrelated. Indeed, to verify that $V_{j}$ and $V_{j+\ell}$ are uncorrelated, note that since $V_{j+\ell} \Perp \hat{S}_{j+1}^{j+\ell}$ and $V_{j+\ell} \Perp V_{j}$, we have $\mathbb{E}\left[\tilde{V}_{j+\ell} \mid V_{j}, \hat{S}_{j+1}^{i}\right]=\mathbf{0}$, and thus

$$
\mathbb{E}\left[\tilde{V}_{i} \tilde{V}_{j+\ell}^{T}\right]=\mathbb{E}\left[\tilde{V}_{i} \mathbb{E}\left[\tilde{V}_{j+\ell}^{T} \mid V_{i}, \hat{S}_{j+1}^{i}\right]\right]=0 .
$$

Using (148) and (150), we write

$$
\begin{aligned}
& \mathbb{E}\left[\left(S_{i}-\mathbb{E}\left[S_{i} \mid \hat{S}^{i}\right]\right)^{T} \mathrm{~L}^{T} \mathrm{~L}\left(S_{i}-\mathbb{E}\left[S_{i} \mid \hat{S}^{i}\right]\right)\right] \\
= & \sum_{j=1}^{i} \mathbb{E}\left[\tilde{V}_{j-1}^{T} \mathrm{~A}^{i-j T} \mathrm{~L}^{T} \mathrm{LA}^{i-j} \tilde{V}_{j-1}\right] \\
\geq & \sum_{j=1}^{i} \mathbb{D}_{\sum_{\ell=0}^{i-j} r_{i-\ell}}\left(\mathrm{LA}^{i-j} V_{j-1}\right) \\
\geq & w N\left(V^{\prime}\right)^{\frac{k}{m}} \sum_{j=1}^{i} a^{2(i-j)} \exp \left(-\frac{2}{n} \sum_{\ell=j}^{i} r_{\ell}\right) \\
\triangleq & w \underline{d}_{i}^{\prime \prime},
\end{aligned}
$$

where (151) uses (148) and (150); (152) leverages Proposition 6 to minimize each term of the sum over $P_{\hat{S}_{j}^{i} \mid V_{j-1}}$ subject to the constraint

$$
I\left(V_{j-1} ; \hat{S}_{j}^{i}\right) \leq \sum_{\ell=j}^{i} r_{\ell},
$$

where $r_{\ell}$ is the per-stage rate, as defined before in (121); (153) is due to Proposition 5. To verify that constraint (121) implies (155), we apply the independence of $V_{j-1}$ and $\hat{S}^{j-1}$ and the chain rule of mutual information to write

$$
I\left(V_{j-1} ; \hat{S}_{j}^{i}\right)=I\left(V_{j-1} ; \hat{S}_{j}^{i} \mid \hat{S}^{j-1}\right) \leq I\left(S^{j} ; \hat{S}_{j}^{i} \mid \hat{S}^{j-1}\right)
$$

$$
=\sum_{\ell=j}^{i} I\left(S^{j} ; \hat{S}_{\ell} \mid \hat{S}^{\ell-1}\right) \leq \sum_{\ell=j}^{i} I\left(S^{\ell} ; \hat{S}_{\ell} \mid \hat{S}^{\ell-1}\right)=\sum_{\ell=j}^{i} r_{\ell} .
$$

Finally, observe using (153) that $\underline{d}_{i+1}^{\prime \prime}$ and $\underline{d}_{i}^{\prime \prime}$ are tied in a recursive relationship akin to that in (129):

$$
\underline{d}_{i+1}^{\prime \prime}=\exp \left(-2 r_{i+1} / n\right)\left(a^{2} \underline{d}_{i}^{\prime \prime}+N\left(V^{\prime}\right)^{\frac{k}{m}}\right) .
$$

The rest of the proof follows along the lines of (130)-(134).

Proof of Theorem 1. If A is rank-deficient, the right side of (14) is $-\infty$, and there is nothing to prove. Assume $\operatorname{det} A \neq$ 0 . Further, the case $\operatorname{rank} \mathrm{B}<n$ implies $\operatorname{det} \mathrm{M}=0$ and is covered by Theorem 3. Assume $\operatorname{rank} \mathrm{B}=n$. According to Corollary 1, Theorem 9 and (59), it suffices to lower bound $\mathbb{R}_{S^{\infty}}(d)$ with weight matrices $\mathbf{W}_{i}$ in (95). Since $\mathbf{S}>0$ (e.g. [57]), it follows that $A^{T} M A>0$ and thus (61) is satisfied with $w=\operatorname{det}\left(\mathrm{A}^{T} \mathrm{MA}\right)$. Therefore, Theorem 9 applies, and the result of Theorem 1 is immediate.

Proof of Theorem 3. According to Corollary 1 and (59), it suffices to lower-bound $\mathbb{R}_{S \infty}(d)$ with weight matrices $\mathrm{W}_{i}$ in (95). Consider first the case rank $\mathrm{B}=n$. Let $\Lambda_{i}$ be a diagonal matrix such that $0 \preceq \Lambda_{i} \preceq J^{T} M_{i}$ J. The weight matrices $J^{T} \mathrm{~A}^{T} \mathrm{M}_{i} \mathrm{AJ}=\mathrm{A}^{\prime T} \mathrm{M}_{i}^{\prime} \mathrm{A}^{\prime} \succeq \mathrm{A}^{\prime T} \Lambda_{i} \mathrm{~A}^{\prime}$, and thus the assumption of Theorem 11 is satisfied with $\vee_{i}=\Lambda_{i}^{\frac{1}{2}} A^{\prime}$, and (23) follows.

If rank $\mathrm{B}<n, \mathrm{M}$ is singular, and the bound in (23) reduces to simply

$$
\mathbb{R}(b) \geq \ell \log a^{\prime} .
$$

To show (158), fix some $\epsilon>0$. Without loss of generality, we assume $\operatorname{rank} \mathrm{B}=m<n$, and we augment $\mathrm{B}$ as follows:

$$
\mathrm{B}_{\epsilon} \triangleq\left[\begin{array}{ll}
\mathrm{B} & \epsilon \tilde{\mathrm{B}}
\end{array}\right] \text {, }
$$

where $(n-m) \times n$ matrix $\tilde{\mathrm{B}}$ is chosen so that the columns of $\mathrm{B}_{\epsilon}$ span $\mathbb{R}^{n}$. We also augment the $m \times m$ matrix $\mathrm{R}$ in (3):

$$
\tilde{\mathrm{R}} \triangleq\left[\begin{array}{cc}
\mathrm{R} & 0 \\
0 & \mathrm{I}_{n-m}
\end{array}\right] \text {. }
$$

Consider the augmented system parameterized by $\epsilon$ :

$$
X_{i+1}=\mathrm{A} X_{i}+\mathrm{B}_{\epsilon} \tilde{U}_{i}+V_{i},
$$

where control inputs $\tilde{U}_{i}$ are $n$-dimensional. The augmented system in (161) achieves the same or smaller quadratic cost as the system in (1), because we can always let $\tilde{U}_{i}=\left[\begin{array}{ll}U_{i} & 0\end{array}\right]^{T}$ to equalize the costs. Therefore,

$$
\mathbb{R}(b) \geq \sup _{\epsilon>0} \mathbb{R}_{\epsilon}(b),
$$

where $\mathbb{R}_{\epsilon}(b)$ denotes the rate-cost function for the system in (161) with parameter $\epsilon$ in (159), (160). In particular, since (158) holds for the augmented system it must also hold for the original system.

Proof of Theorem 4. According to Proposition 6, the causal rate-distortion function of the uncontrolled process $\left\{S_{i}\right\}$ with weight matrices $\mathrm{W}_{i}=\mathrm{A}^{T} \mathrm{M}_{i} \mathrm{~A}$ is equal to that of $\left\{\mathrm{A} S_{i}\right\}$ with weight matrices $\left\{\mathrm{M}_{i}\right\}$. Putting $S_{i}^{\prime \prime} \triangleq \mathrm{A} S_{i}$ and noticing that $S_{i+1}^{\prime \prime}=\mathrm{A} S_{i}^{\prime \prime}+\mathrm{A} V_{i}$, we apply Theorem 12 to conclude

$$
\mathbb{R}_{S^{\prime \prime \infty}}(d) \geq \frac{m}{2} \log \left(a^{2}+\frac{\mu N(\mathrm{~A} V)^{\frac{n}{m}}}{d / m}\right),
$$


Proof of Theorem 5. We assume $\operatorname{rank} \mathrm{B}=\operatorname{rank} \mathrm{C}=n$. The more general case is considered in Theorem 7.

According to (86) and (90), $\left\{\hat{X}_{i}\right\}$ is Gauss-Markov process, whose additive noise $\mathrm{K}_{i} \tilde{Y}_{i}$ has covariance matrix $\mathrm{N}_{i} \triangleq$ $\mathrm{K}_{i}\left(\mathrm{CP}_{i \mid i-1} \mathrm{C}^{T}+\Sigma_{W}\right) \mathrm{K}_{i}^{T}$. By Theorem 9 and (59),

$$
\mathbb{R}_{\hat{X}^{\infty} \| \mathcal{D} U^{\infty}}(d) \geq \log |\operatorname{det} \mathrm{A}|+\frac{n}{2} \log \left(1+\frac{(\operatorname{det} \mathrm{NM})^{\frac{1}{n}}}{d / n}\right),
$$

and Theorem 5 follows immediately via Corollary 1 .

Proof of Theorem 7. The proof is similar to that of Theorem 3 and uses Theorem 11 to lower-bound $\mathbb{R}_{\hat{X}^{\infty} \| \mathcal{D} U^{\infty}}(d)$.

Proof of Theorem 8. The proof is similar to that of Theorem 4, and uses Theorem 12 to lower-bound $\mathbb{R}_{\hat{X} \infty \| \mathcal{D} U^{\infty}}(d)$.

\section{ACHIEVABILITY THEOREMS: TOOLS AND PROOFS}

In this section, we will prove Theorems 2 and 6.

Our achievability scheme employs lattice quantization. A lattice $\mathcal{C}$ in $\mathbb{R}^{n}$ is a discrete set of points that is closed under reflection and addition. The nearest-neighbor quantizer is the mapping $q_{\mathcal{C}}: \mathbb{R}^{n} \mapsto \mathcal{C}$ defined by

$$
\mathbf{q}_{\mathcal{C}}(x) \triangleq \underset{c \in \mathcal{C}}{\operatorname{argmin}}\|x-c\| .
$$

Covering efficiency of lattice $\mathcal{C}$ is measured by

$$
\rho_{\mathcal{C}} \triangleq\left(\frac{B_{\mathcal{C}}}{V_{\mathcal{C}}}\right)^{\frac{1}{n}}
$$

where $V_{\mathcal{C}}$ the volume of the Voronoi cells of lattice $\mathcal{C}$ :

$$
V_{\mathcal{C}} \triangleq \operatorname{Vol}\left(\left\{x \in \mathbb{R}^{n}: \mathbf{q}_{\mathcal{C}}(x)=c\right\}\right),
$$

where arbitrary $c \in \mathcal{C}$, and $B_{\mathcal{C}}$ is the volume of a ball whose radius is equal to that of the Voronoi cells of $\mathcal{C}$. The radius of $B_{\mathcal{C}}$ is called covering radius of lattice $\mathcal{C}$. By definition, $\rho_{\mathcal{C}} \geq 1$, and the closer $\rho_{\mathcal{C}}$ is to 1 the more sphere-like the Voronoi cells of $\mathcal{C}$ are and the better lattice $\mathcal{C}$ is for covering.

Proof of Theorem 10. The proof analyses a DPCM scheme. First, we describe how the codebook is generated, then we describe the operation of the encoder and the decoder, and then we proceed to the analysis of the scheme.

Codebook design. To maximize covering efficiency, we use the best known $n$-dimensional lattice quantizer $\mathrm{q}=\mathrm{q}_{\mathcal{C}^{n}}$ scaled so that its covering radius is $\leq \sqrt{d}$.

Encoder. Upon observing $S_{i}$, the encoder computes the state innovation $\tilde{S}_{i}$ recursively using the formula

$$
\tilde{S}_{i} \triangleq S_{i}-\mathrm{A} \hat{S}_{i-1},
$$

where $\hat{S}_{i}$ is the decoder's state estimate at time $i$ (put $\hat{S}_{0} \triangleq 0$ ). The encoder transmits the index of

$$
Q_{i} \triangleq \mathrm{q}\left(\mathrm{W}_{i}^{\frac{1}{2}} \tilde{S}_{i}\right) .
$$

Decoder. The decoder recovers the lattice cell identified by the encoder, and forms its state estimate as

$$
\begin{aligned}
& \hat{S}_{i}=\mathrm{A} \hat{S}_{i-1}+\hat{\tilde{S}}_{i}, \\
& \hat{\tilde{S}}_{i} \triangleq \mathrm{W}_{i}^{-\frac{1}{2}} Q_{i} .
\end{aligned}
$$

Analysis. The distortion at step $i$ is given by

$$
\left(S_{i}-\hat{S}_{i}\right)^{T} \mathrm{~W}_{i}\left(S_{i}-\hat{S}_{i}\right)=\left(\tilde{S}_{i}-\hat{\tilde{S}}_{i}\right)^{T} \mathrm{~W}_{i}\left(\tilde{S}_{i}-\hat{\tilde{S}}_{i}\right)
$$

$$
=\left\|\mathrm{W}_{i}^{\frac{1}{2}} \tilde{S}_{i}-Q_{i}\right\|^{2} \leq d .
$$

It remains to upper-bound the entropy of $Q^{t}$. Since $H\left(Q^{t}\right) \leq$ $\sum_{i=1}^{t} H\left(Q_{i}\right)$, it suffices to bound the unconditional entropy of $Q_{i}$. First, we establish that $\mathrm{W}_{i}^{\frac{1}{2}} \tilde{S}_{i}$ has a regular density. Using the assumption that $V_{i}$ has a $\left(c_{0}, c_{1}\right)$-regular density, it's easy to see that $\mathrm{W}_{i}^{\frac{1}{2}} V_{i}$ has $\left(w_{i}^{-1} c_{0}, w_{i}^{-1} c_{1}\right)$-regular density, where $w_{i}$ is the minimum eigenvalue of $\mathbf{W}_{i}$. Furthermore, similar to (171),

$$
\left(S_{i}-\hat{S}_{i}\right)^{T} \mathrm{~A}^{T} \mathrm{~W}_{i} \mathrm{~A}\left(S_{i}-\hat{S}_{i}\right) \leq a_{i} d,
$$

where $a_{i}$ is the following operator norm of $\mathrm{A}$ :

$$
a_{i} \triangleq \sup _{z \neq \mathbf{0}} \frac{z^{T} \mathrm{~A}^{T} \mathrm{~W}_{i} \mathrm{~A} z}{z^{T} \mathrm{~W}_{i} z} .
$$

From (167) and (58),

$$
\tilde{S}_{i}=\mathrm{A}\left(S_{i-1}-\hat{S}_{i-1}\right)+V_{i-1},
$$

and it follows via [46, Prop. 3] that $\mathrm{W}_{i}^{\frac{1}{2}} \tilde{S}_{i}$ has $\left(w_{i}^{-1}\left(c_{0}+\right.\right.$ $\left.\left.a_{i}^{\frac{1}{2}} d^{\frac{1}{2}} c_{1}\right), w_{i}^{-1} c_{1}\right)$-regular density.

Combining (172) and (174) yields

$$
\operatorname{Var}\left[\mathrm{W}_{i}^{\frac{1}{2}} \tilde{S}_{i}\right] \leq a_{i} d+v_{i}
$$

where we denoted for brevity

$$
v_{i} \triangleq \operatorname{tr}\left(\Sigma_{V} \mathrm{~W}_{i}\right)
$$

Now, [44, Th. 8] implies that the entropy of $Q_{i}$ satisfies:

$$
\begin{aligned}
& H\left(Q_{i}\right) \leq \min _{\tilde{d} \leq d}\left\{\frac{n}{2} \log \frac{N\left(\mathrm{~W}_{i}^{\frac{1}{2}} \tilde{S}_{i}\right)}{\tilde{d} / n}\right. \\
& \left.+2 \frac{\tilde{d}^{\frac{1}{2}}}{w_{i}} \log e \cdot\left(c_{1}\left(a_{i} d+v_{i}\right)^{\frac{1}{2}}+c_{0}+c_{1}\left(1+a_{i}^{\frac{1}{2}}\right) \tilde{d}^{\frac{1}{2}}\right)\right\} \\
& +\alpha_{n}+n \log \rho_{\mathcal{C}_{n}},
\end{aligned}
$$

where $\alpha_{n}$ and $n \log \rho_{\mathcal{C}_{n}}$ are of order $O(\log n)$.

To estimate the entropy power of $\mathrm{W}_{i}^{\frac{1}{2}} \tilde{S}_{i}$, we use (172) and (174) to bound the Wasserstein distance between $\mathrm{W}_{i}^{\frac{1}{2}} \tilde{S}_{i}$ and $\mathrm{W}_{i}^{\frac{1}{2}} V_{i-1}$, so that [46, Prop. 1] applies to yield:

$$
\begin{aligned}
h\left(\mathrm{~W}_{i}^{\frac{1}{2}} \tilde{S}_{i}\right) \leq & h\left(\mathrm{~W}_{i}^{\frac{1}{2}} V_{i-1}\right)+\log e \frac{\left(a_{i} d_{i}\right)^{\frac{1}{2}}}{w_{i}} \\
& \cdot\left(\frac{c_{1}}{2} v_{i}^{\frac{1}{2}}+\frac{c_{1}}{2}\left(a_{i} d+v_{i}\right)^{\frac{1}{2}}+c_{0}\right) .
\end{aligned}
$$

Combining (177) and (178), we conclude that

$$
\begin{aligned}
& H\left(Q_{i}\right) \leq \\
& \min _{\tilde{d} \leq d}\left\{\frac{n}{2} \log \frac{N\left(\mathrm{~W}_{i}^{\frac{1}{2}} V_{i-1}\right)}{\tilde{d} / n}+\beta_{i}(\tilde{d})\right\}+\alpha_{n}+n \log \rho_{\mathcal{C}_{n}},
\end{aligned}
$$

where $\beta_{i}(d)=O\left(d^{\frac{1}{2}}\right)$ is given by

$$
\begin{aligned}
\beta_{i}(d) \triangleq & \frac{d^{\frac{1}{2}}}{w_{i}} \log e\left(\frac{1}{2} c_{1} a_{i}^{\frac{1}{2}} v_{i}^{\frac{1}{2}}+c_{0}\left(2+a_{i}^{\frac{1}{2}}\right)\right. \\
& \left.+c_{1}\left(2+\frac{a_{i}^{\frac{1}{2}}}{2}\right)\left(a_{i} d+v_{i}\right)^{\frac{1}{2}}+2 c_{1}\left(1+a_{i}^{\frac{1}{2}}\right) d^{\frac{1}{2}}\right) .
\end{aligned}
$$

Recalling (103) and using the resulting bound (179) to bound $\lim _{t \rightarrow \infty} \frac{1}{t} \sum_{i=1}^{t} H\left(Q_{i}\right)$, we obtain the statement of Theorem 10. 
Proof of Theorem 2. Due to Corollary 1 and (60), it suffices to bound the entropy-distortion function of the process (58). Such a bound is provided in Theorem 10 .

Proof of Theorem 6. Due to Corollary 1, it suffices to bound the conditional entropy-distortion function of the Kalman filter estimates process in (86). Such a bound follows from (60) and Theorem 10.

\section{CONCLUSION}

We studied the fundamental tradeoff between the communication requirements and the attainable quadratic cost in fully and partially observed linear stochastic control systems. We introduced the rate-cost function in Definition 1, and showed sharp lower bounds to it in Theorems 1, 3, 4 (fully observed system) and Theorems 5, 7, 8 (partially observed system). The achievability results in Theorem 2 (fully observed system) and Theorem 6 (partially observed system) show that the converse can be approached, in the high rate / low cost regime, by a simple variable-rate lattice-based scheme in which only the quantized value of the innovation is transmitted. Via the separation principle, the same conclusions hold for causal compression of Markov sources: a converse, which may be viewed as a causal counterpart of Shannon's lower bound, is stated in Theorem 9, and a matching achievability in Theorem 10.

Extending the analysis of the partially observed case to nonGaussian noises would be of interest. It also remains an open question whether the converse bound in Theorem 1 can be approached by fixed-rate quantization, or over noisy channels. Finally, it would be interesting to see whether using non-lattice quantizers can help to narrow down the gap in Fig. 3.

\section{ACKNOWLEDGEMENT}

The authors acknowledge many stimulating discussions with Dr. Anatoly Khina and his helpful comments on the earlier versions of the manuscript. The authors are also grateful to Ayush Pandey, who generated the plot in Fig. 3.

\section{REFERENCES}

[1] V. Kostina and B. Hassibi, "Rate-cost tradeoffs in control," in Proceedings 54th Annual Allerton Conference on Communication, Control and Computing, Monticello, IL, Oct. 2016, pp. 1157-1164.

[2] S. Tatikonda, A. Sahai, and S. Mitter, "Stochastic linear control over a communication channel," IEEE Transactions on Automatic Control, vol. 49, no. 9, pp. 1549-1561, 2004.

[3] J. Massey, "Causality, feedback and directed information," in Proc. Int Symp. Inf. Theory Applic.(ISITA-90), 1990, pp. 303-305.

[4] G. Kramer, "Directed information for channels with feedback," Ph.D. dissertation, ETH Zurich, 1998.

[5] M. Gastpar, B. Rimoldi, and M. Vetterli, "To code, or not to code: lossy source-channel communication revisited," IEEE Transactions on Information Theory, vol. 49, no. 5, pp. 1147-1158, May 2003.

[6] J. Baillieul, "Feedback designs for controlling device arrays with communication channel bandwidth constraints," in ARO Workshop on Smart Structures, Pennsylvania State Univ, 1999, pp. 16-18.

[7] W. S. Wong and R. W. Brockett, "Systems with finite communication bandwidth constraints. II. Stabilization with limited information feedback," IEEE Transactions on Automatic Control, vol. 44, no. 5, pp. 1049-1053, 1999.

[8] S. Tatikonda and S. Mitter, "Control under communication constraints," IEEE Transactions on Automatic Control, vol. 49, no. 7, pp. 1056-1068, 2004.
[9] B. G. N. Nair, F. Fagnani, S. Zampieri, and R. J. Evans, "Feedback control under data rate constraints: An overview," Proceedings of the IEEE, vol. 95, no. 1, pp. 108-137, 2007.

[10] G. N. Nair and R. J. Evans, "Stabilizability of stochastic linear systems with finite feedback data rates," SIAM Journal on Control and Optimization, vol. 43, no. 2, pp. 413-436, 2004.

[11] R. W. Brockett and D. Liberzon, "Quantized feedback stabilization of linear systems," IEEE transactions on Automatic Control, vol. 45, no. 7, pp. 1279-1289, 2000.

[12] S. Yüksel, "Stochastic stabilization of noisy linear systems with fixedrate limited feedback," IEEE Transactions on Automatic Control, vol. 55, no. 12 , pp. $2847-2853,2010$.

[13] _ "Jointly optimal LQG quantization and control policies for multidimensional systems," IEEE Transactions on Automatic Control, vol. 59, no. 6, pp. 1612-1617, 2014.

[14] H. S. Witsenhausen, "On the structure of real-time source coders," The Bell System Technical Journal, vol. 58, no. 6, pp. 1437-1451, 1979.

[15] N. Gaarder and D. Slepian, "On optimal finite-state digital transmission systems," IEEE Transactions on Information Theory, vol. 28, no. 2, pp. $167-186,1982$.

[16] J. C. Walrand and P. Varaiya, "Optimal causal coding-decoding problems," IEEE Transactions on Information Theory, vol. 29, no. 6, pp. 814-820, 1983.

[17] V. S. Borkar, S. K. Mitter, and S. Tatikonda, "Optimal sequential vector quantization of Markov sources," SIAM journal on control and optimization, vol. 40, no. 1, pp. 135-148, 2001.

[18] D. Teneketzis, "On the structure of optimal real-time encoders and decoders in noisy communication," IEEE Transactions on Information Theory, vol. 52, no. 9, pp. 4017-4035, 2006.

[19] T. Linder and S. Yüksel, "On optimal zero-delay coding of vector Markov sources," IEEE Transactions on Information Theory, vol. 60 no. 10, pp. 5975-5991, 2014

[20] R. G. Wood, T. Linder, and S. Yüksel, "Optimality of Walrand-Varaiya type policies and approximation results for zero delay coding of Markov sources," in Proceedings 2015 IEEE International Symposium on Information Theory, Hong Kong, June 2015.

[21] S. Yüksel, T. Basar, and S. P. Meyn, "Optimal causal quantization of Markov sources with distortion constraints," in Information Theory and Applications Workshop, 2008, 2008, pp. 26-30.

[22] N. Elia and S. K. Mitter, "Stabilization of linear systems with limited information," IEEE transactions on Automatic Control, vol. 46, no. 9, pp. 1384-1400, 2001.

[23] A. Gorbunov and M. S. Pinsker, "Prognostic epsilon entropy of a Gaussian message and a Gaussian source," Problemy Peredachi Informatsii, vol. 10, no. 2, pp. 5-25, 1974

[24] C. D. Charalambous, P. A. Stavrou, and N. U. Ahmed, "Nonanticipative rate distortion function and relations to filtering theory," IEEE Transactions on Automatic Control, vol. 59, no. 4, pp. 937-952, 2014.

[25] C. D. Charalambous and A. Farhadi, "LQG optimality and separation principle for general discrete time partially observed stochastic systems over finite capacity communication channels," Automatica, vol. 44, no. 12 , pp. 3181-3188, 2008

[26] C. D. Charalambous, C. K. Kourtellaris, and C. Hadjicostis, "Optimal encoder and control strategies in stochastic control subject to rate constraints for channels with memory and feedback," in 2011 50th IEEE Conference on Decision and Control and European Control Conference, Dec 2011, pp. 4522-4527.

[27] E. Shafieepoorfard, M. Raginsky, and S. P. Meyn, "Rationally inattentive control of Markov processes," SIAM Journal on Control and Optimization, vol. 54, no. 2, pp. 987-1016, 2016.

[28] E. I. Silva, M. S. Derpich, and J. Ostergaard, "A framework for control system design subject to average data-rate constraints," IEEE Transactions on Automatic Control, vol. 56, no. 8, pp. 1886-1899, 2011.

[29] E. Silva, M. Derpich, J. Ostergaard, and M. Encina, "A characterization of the minimal average data rate that guarantees a given closed-loop performance level," IEEE Transactions on Automatic Control, 2016.

[30] T. Tanaka, K. H. Johansson, T. Oechtering, H. Sandberg, and M. Skoglund, "Rate of prefix-free codes in LQG control systems," in Proceedings 2016 IEEE International Symposium on Information Theory, Barcelona, Spain, July 2016, pp. 2399-2403.

[31] P. A. Stavrou, J. Østergaard, C. D. Charalambous, and M. Derpich, "An upper bound to zero-delay rate distortion via kalman filtering for vector gaussian sources," in Proceedings 2017 IEEE Information Theory Workshop (ITW), Nov 2017, pp. 534-538.

[32] M. S. Derpich and J. Ostergaard, "Improved upper bounds to the causal quadratic rate-distortion function for Gaussian stationary sources," IEEE 
Transactions on Information Theory, vol. 58, no. 5, pp. 3131-3152, May 2012.

[33] T. Tanaka, K.-K. K. Kim, P. A. Parrilo, and S. K. Mitter, "Semidefinite programming approach to Gaussian sequential rate-distortion trade-offs," IEEE Transactions on Automatic Control, vol. 62, no. 4, pp. 1896-1910, 2017.

[34] T. Tanaka, P. M. Esfahani, and S. K. Mitter, "LQG control with minimum directed information: Semidefinite programming approach," IEEE Transactions on Automatic Control, 2017.

[35] C. E. Shannon, "Coding theorems for a discrete source with a fidelity criterion," IRE Int. Conv. Rec., vol. 7, no. 1, pp. 142-163, Mar. 1959 reprinted with changes in Information and Decision Processes, R. E. Machol, Ed. New York: McGraw-Hill, 1960, pp. 93-126.

[36] _ - "A mathematical theory of communication," Bell Syst. Tech. J., vol. 27, pp. 379-423, 623-656, July and October 1948.

[37] A. J. Stam, "Some inequalities satisfied by the quantities of information of Fisher and Shannon," Information and Control, vol. 2, no. 2, pp. 101-112, 1959.

[38] H. Gish and J. Pierce, "Asymptotically efficient quantizing," IEEE Transactions on Information Theory, vol. 14, no. 5, pp. 676-683, 1968

[39] J. Ziv, "On universal quantization," IEEE Transactions on Information Theory, vol. 31, no. 3, pp. 344-347, 1985.

[40] R. Zamir and M. Feder, "On lattice quantization noise," IEEE Transactions on Information Theory, vol. 42, no. 4, pp. 1152-1159, Jul. 1996.

[41] A. Gersho, "Asymptotically optimal block quantization," IEEE Transactions on Information Theory, vol. 25, no. 4, pp. 373-380, Jul. 1979.

[42] R. Zamir and M. Feder, "On universal quantization by randomized uniform/lattice quantizers," IEEE Transactions on Information Theory, vol. 38, no. 2, pp. 428-436, Mar. 1992.

[43] T. T. Linder and K. K. Zeger, "Asymptotic entropy-constrained performance of tessellating and universal randomized lattice quantization," IEEE Transactions on Information Theory, vol. 40, no. 2, pp. 575-579, Mar. 1994.

[44] V. Kostina, "Data compression with low distortion and finite blocklength," IEEE Transactions on Information Theory, vol. 63, no. 7, pp. 4268-4285, July 2017.

[45] M. Fu, "Lack of separation principle for quantized linear quadratic Gaussian control," IEEE Transactions on Automatic Control, vol. 57, no. 9, pp. 2385-2390, 2012.

[46] Y. Polyanskiy and Y. Wu, "Wasserstein continuity of entropy and outer bounds for interference channels," IEEE Transactions on Information Theory, vol. 62, no. 7, pp. 3992-4002, July 2016.

[47] Y.-H. Kim, "A coding theorem for a class of stationary channels with feedback," IEEE Transactions on Information Theory, vol. 54, no. 4, pp. 1488-1499, 2008.

[48] S. Tatikonda and S. Mitter, "The capacity of channels with feedback," IEEE Transactions on Information Theory, vol. 55, no. 1, pp. 323-349, Jan 2009.

[49] R. Bansal and T. Başar, "Simultaneous design of measurement and control strategies for stochastic systems with feedback," Automatica, vol. 25, no. 5, pp. 679-694, 1989.

[50] J. S. Freudenberg, R. H. Middleton, and V. Solo, "Stabilization and disturbance attenuation over a Gaussian communication channel," IEEE Transactions on Automatic Control, vol. 55, no. 3, pp. 795-799, 2010.

[51] A. Khina, G. M. Pettersson, V. Kostina, and B. Hassibi, "Multi-rate control over AWGN channels: An analog joint source-channel coding perspective," in 55th IEEE Conference on Decision and Control. Las Vegas, NV, Dec. 2016.

[52] A. Wyner, "An upper bound on the entropy series," Information and Control, vol. 20, no. 2, pp. 176-181, Mar. 1972.

[53] N. Alon and A. Orlitsky, "A lower bound on the expected length of one-to-one codes," IEEE Transactions on Information Theory, vol. 40, no. 5, pp. 1670-1672, Sep. 1994.

[54] W. Szpankowski and S. Verdú, "Minimum expected length of fixedto-variable lossless compression without prefix constraints: memoryless sources," IEEE Transactions on Information Theory, vol. 57, no. 7, pp. 4017-4025, July 2011.

[55] T. Tanaka, "Semidefinite representation of sequential rate-distortion function for stationary Gauss-Markov processes," in Proceedings 2015 IEEE Conference on Control Applications (CCA), Sep. 2015, pp. 12171222.

[56] T. Fischer, "Optimal quantized control," IEEE Transactions on Automatic Control, vol. 27, no. 4, pp. 996-998, Aug 1982.

[57] D. P. Bertsekas, Dynamic programming and optimal control. Athena Scientific Belmont, MA, 1995, vol. 1.

[58] S. Tatikonda, "Control under communication constraints," Ph.D. dissertation, M.I.T., 2000.
[59] T. M. Cover and J. A. Thomas, Elements of information theory, 2nd ed. John Wiley \& Sons, 2012.

[60] A. Gerrish and P. Schultheiss, "Information rates of non-Gaussian processes," IEEE Transactions on Information Theory, vol. 10, no. 4, pp. 265-271, Oct. 1964.

[61] Y. N. Linkov, "Evaluation of $\epsilon$-entropy of random variables for small $\epsilon$," Problems of Information Transmission, vol. 1, no. 2, pp. 18-26, 1965.

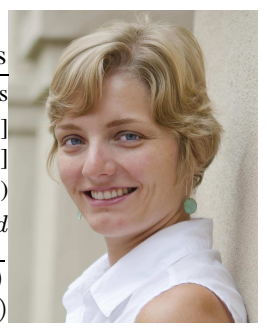

Victoria Kostina joined Caltech as an Assistant Professor of Electrical Engineering in the fall of 2014. She holds a Bachelor's degree from Moscow institute of Physics and Technology (2004), where she was affiliated with the Institute for Information Transmission Problems of the Russian Academy of Sciences, a Master's degree from University of Ottawa (2006), and a PhD from Princeton University (2013). She received the Natural Sciences and Engineering Research Council of Canada postgraduate scholarship (2009-2012), the Princeton Electrical Engineering Best Dissertation Award (2013), the Simons-Berkeley research fellowship (2015) and the NSF CAREER award (2017). Kostina's research spans information theory, coding, control and communications.

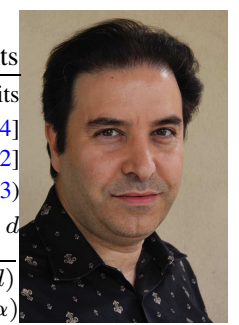

Babak Hassibi was born in Tehran, Iran, in 1967 He received the B.S. degree from the University of Tehran in 1989, and the M.S. and Ph.D. degrees from Stanford University in 1993 and 1996, respectively, all in electrical engineering.

He has been with the California Institute of Technology since January 2001, where he is currently the Mose and Lilian S. Bohn Professor of Electrical Engineering. From 2013-2016 he was the Gordon M. Binder/Amgen Professor of Electrical Engineering and from 2008-2015 he was Executive Officer of Electrical Engineering, as well as Associate Director of Information Science and Technology. From October 1996 to October 1998 he was a research associate at the Information Systems Laboratory, Stanford University, and from November 1998 to December 2000 he was a Member of the Technical Staff in the Mathematical Sciences Research Center at Bell Laboratories, Murray Hill, NJ. He has also held short-term appointments at Ricoh California Research Center, the Indian Institute of Science, and Linkoping University, Sweden. His research interests include communications and information theory, control and network science, and signal processing and machine learning. $\mathrm{He}$ is the coauthor of the books (both with A.H. Sayed and T. Kailath) Indefinite Quadratic Estimation and Control: A Unified Approach to $H^{2}$ and $H^{\infty}$ Theories (New York: SIAM, 1999) and Linear Estimation (Englewood Cliffs, NJ: Prentice Hall, 2000). He is a recipient of an Alborz Foundation Fellowship, the 1999 O. Hugo Schuck best paper award of the American Automatic Control Council (with H. Hindi and S.P. Boyd), the 2002 National ScienceFoundation Career Award, the 2002 Okawa Foundation Research Grant for Information and Telecommunications, the 2003 David and Lucille Packard Fellowship for Science and Engineering, the 2003 Presidential Early Career Award for Scientists and Engineers (PECASE), and the 2009 Al-Marai Award for Innovative Research in Communications, and was a participant in the 2004 National Academy of Engineering "Frontiers in Engineering"program.

He has been a Guest Editor for the IEEE Transactions on Information Theory special issue on "space-time transmission, reception, coding and signal processing" was an Associate Editor for Communications of the IEEE Transactions on Information Theory during 2004-2006, and is currently an Editor for the Journal "Foundations and Trends in Information and Communication" and for the IEEE Transactions on Network Science and Engineering. He is an IEEE Information Theory Society Distinguished Lecturer for 2016-2017. 


\section{APPENDIX}

This appendix summarizes the tools used in the proofs of Section VI. The first is a tool to bound the difference between the differential entropies of two random vectors whose distributions are close to each other.

Proposition 8 ([46, Prop. 1]). Let $X$ and $Y$ be random vectors with finite second moments. If the density of $X$ is $\left(c_{0}, c_{1}\right)$ regular, then

$$
\begin{aligned}
h(Y)-h(X) & \leq \log e\left(\frac{c_{1}}{2}\left(\mathbb{E}\left[\|X\|^{2}\right]\right)^{\frac{1}{2}}\right. \\
& \left.+\frac{c_{1}}{2}\left(\mathbb{E}\left[\|Y\|^{2}\right]\right)^{\frac{1}{2}}+c_{0}\right) W(X, Y),
\end{aligned}
$$

where $W(X, Y)$ is the Wasserstein distance between the distributions of $X$ and $Y$ :

$$
W(X, Y) \triangleq \inf \left(\mathbb{E}\left[\|X-Y\|^{2}\right]\right)^{\frac{1}{2}},
$$

where the infimum is over all joint distributions $P_{X Y}$ whose marginals are $P_{X}$ and $P_{Y}$.

The next result helps us establish that the random vectors encountered at each step of the control system operation have regular densities.

Proposition 9 ( [46, Prop. 3]). If the density of $Z$ is $\left(c_{0}, c_{1}\right)$ regular and $B \Perp Z,\|B\| \leq b$ a.s., then that of $B+Z$ is $\left(c_{0}+c_{1} b, c_{1}\right)$-regular.

The next result gives an upper bound to the output entropy of lattice quantizers.

Theorem 16 (Corollary to [44, Th. 8]). Suppose that $f_{X}$ is $\left(c_{0}, c_{1}\right)$-regular. There exists a lattice quantizer $\mathrm{q}=\mathrm{q}_{\mathcal{C}^{n}}$ such that

$$
\sup _{x \in \mathbb{R}^{n}}\|x-\mathrm{q}(x)\|^{2} \leq d
$$

and

$$
\begin{aligned}
H(q(X)) \leq & \min _{\tilde{d} \leq d}\left(\frac{n}{2} \log \frac{N(X)}{\tilde{d} / n}+\alpha_{n}+n \log \rho_{\mathcal{C}_{n}}\right. \\
& \left.+2 \tilde{d}^{\frac{1}{2}} \log e\left(c_{1} \sqrt{\operatorname{Var}[X]}+c_{0}+c_{1} \tilde{d}^{\frac{1}{2}}\right)\right),
\end{aligned}
$$

where $\rho_{\mathcal{C}_{n}}$ is the lattice covering efficiency defined in (165),

$$
\alpha_{n} \triangleq \frac{n}{2} \log \frac{2 e}{n}+\log \Gamma\left(\frac{n}{2}+1\right),
$$

and $\Gamma(\cdot)$ is the Gamma function.

The leading term in (184) is Shannon's lower bound (the functional inverse of (115)). The contribution of the remaining terms becomes negligible if $n$ is large and $d$ is small. Indeed, by Stirling's approximation, as $n \rightarrow \infty$,

$$
\alpha_{n}=\frac{1}{2} \log n+O(1) .
$$

On the other hand, Rogers [62, Theorem 5.9] showed that for each $n \geq 3$, there exists an $n$-dimensional lattice $\mathcal{C}_{n}$ with covering efficiency

$$
n \log \rho_{\mathcal{C}_{n}} \leq \log _{2} \sqrt{2 \pi e}(\log n+\log \log n+c),
$$

where $c$ is a constant. Therefore, the terms $n \log \rho_{\mathcal{C}_{n}}$ and $\alpha_{n}$ are logarithmic in $n$, so in high dimension their contribution becomes negligible compared to the first term in (184).
In low dimension, the contribution of these terms can be computed as follows. The thinnest lattice covering is known in dimensions 1 to 23 is Voronoi's principal lattice of the first type [63] $\left(A_{n}^{*}\right)$, which has covering efficiency

$$
\rho_{A_{n}^{*}}=\frac{\pi^{\frac{1}{2}}(n+1)^{\frac{1}{2 n}}}{\left(\Gamma\left(\frac{n}{2}+1\right)\right)^{\frac{1}{n}}} \sqrt{\frac{n(n+2)}{12(n+1)}} .
$$

$A_{n}^{*}$ is proven to be the thinnest lattice covering possible in dimensions $n=1,2, \ldots, 5$. For $A_{n}^{*}$-based lattice quantizer, we can compute the constant appearing in (184) as

$$
\log \rho_{A_{n}^{*}}+\frac{\alpha_{n}}{n}=\frac{1}{2} \log \frac{2 \pi e(n+2)}{12(n+1)^{1-\frac{1}{n}}} .
$$

\section{APPENDIX REFERENCES}

[62] C. A. Rogers, Packing and covering. Cambridge University Press, 1964, no. 54.

[63] J. H. Conway and N. J. A. Sloane, Sphere packings, lattices and groups. Springer Science \& Business Media, New York, 2013, vol. 290. 